\title{
Longitudinal Vehicle State Estimation Using Nonlinear and Parameter-Varying Observers
}

\author{
E. Hashemi, S. Khosravani, A. Khajepour, A. Kasaiezadeh, S. K. Chen, and B. Litkouhi
}

\begin{abstract}
A corner-based velocity estimation approach is proposed which is used for vehicle's traction and stability control systems. This approach incorporates internal tire states within the vehicle kinematics and enables the velocity estimator to work for a wide range of maneuvers without road friction information. Tire models have not been widely implemented in velocity estimators because of uncertain road friction and varying tire parameters, but the current study utilizes a simplified LuGre model with the minimum number of tire parameters and estimates velocity robust to model uncertainties. The proposed observer uses longitudinal forces, updates the states by minimizing the longitudinal force estimation error, and provides accurate outcomes at each tire. The estimator structure is shown to be robust to road conditions and rejects disturbances and model uncertainties effectively. Taking into account the vehicle dynamics is time-varying, the stability of the observer for the linear parameter varying model is proved, time-varying observer gains are designed, and the performance is studied. Road test experiments have been conducted and the results are used to validate the proposed approach.
\end{abstract}

\section{INTRODUCTION}

$\mathbf{V}$ EHICLE control systems require lateral and longitudinal states (velocities and forces) to control wheel slip, vehicle yaw rates, and side slip angles. Among these states, longitudinal state estimation makes major contributions into vehicle stability and traction control. Recent literature has adopted two fundamental approaches regarding longitudinal velocity estimation. One is the modified kinematic-based approach [1], which uses acceleration and the yaw rate measurements from an inertial measurement unit (IMU) and estimates the vehicle states employing stochastic estimators such as Kalman. This method does not employ a tire model, but instead usually utilizes GPS receiver to remove estimation bias. Bevly et al. proposed an estimation method in [2], [3] using a singleantenna GPS and measurements from IMU. Integrating the yaw rate during turning, their method obtains the vehicle heading. The state estimation structure provided by Ryu and Gerdes in [4] is a practical approach for determining vehicle states in which integration of the inertial sensors is performed when GPS data is unavailable. A full description of the planar vehicle dynamics is also implemented in their work to estimate lateral states using the yaw angle obtained by a GPS. However, these tire-free approaches rely on accurate GPS data which

Ehsan Hashemi, Saeid Khosravani, and Amir Khajepour are with the Department of Mechanical and Mechatronics Engineering, University of Waterloo, ON, Canada. email: ehashemi, saeid.khosravani.1, a.khajepour@uwaterloo.ca

Alireza Kasaiezadeh, Shih-Ken Chen, and Bakhtiar Litkouhi are with the R\&D Department, General Motors Co., Warren, MI, USA may be lost. It also imposes additional high costs on production vehicles.

The other longitudinal velocity estimation method exploits an observer on vehicle's longitudinal dynamics with the tire model. The advantage of this method is that it considers the tire capacities, although it still needs road conditions and tire parameters, which may vary significantly in different driving conditions. Using a linear Kalman filter, together with the fuzzy logic approach, Kobayashi et al. proposed a state estimator in [5], which exhibits acceptable performance and low computational loads. To deal with the varying tire parameters and model uncertainties, tire slips are used to define a model scheduling in [6], [7]. Nonlinear observers are studied on bicycle model in [8], [9] for vehicle state estimation. An Extended Kalman filter (EKF) is employed for both longitudinal and lateral vehicle state estimation in [10], [11]. EKF has also been used in [12], [13] along with the Burckhardt model [14] to estimate the vehicle states and parameters of the tire model; an EKF with smooth variable structure is also utilized in [15]. Computational complexities of the EKF justify using a reliable approach such as the unscented Kalman filter (UKF) [16], [17] without any need for linearization in system dynamics. Nonlinear stochastic estimation capability of the UKF provides acceptable numerical efficiency compared with the EKF. Antonov et al. [18] employed an UKF for vehicle state estimation and provided a longitudinal/lateral velocity estimator at each corner. They utilized wheel torques, wheel speeds at each corner, and a simplified empirical Magic formula [19] as the tire model. However, this method needs the road condition and is sensitive to the effective tire rolling radius because it uses the slip ratio. Wielitzka et al. presented a method in [20] for the vehicle state estimation using UKF, but their approach employs tire model that needs road friction.

On the other hand, to tackle the unknown road condition issue, other approaches estimate vehicle states as well as the road friction [21]. A sliding-mode observer is proposed in [22] based on the LuGre dynamic friction model to estimate longitudinal velocity as well as the friction limit. However, concurrent estimation of the road friction for low excitation and low-slip regions is challenging. Li et al. used nonlinear observer and the Dugoff tire model in [23] for the vehicle state estimation, but their method necessitates steering torque measurement for identification of the tire model's friction parameter. A nonlinear model and a gain scheduling scheme is considered in [24] on the linear parameter-varying observer to cope with the road friction changes. Zhang et al. presented a different version of the sliding-mode observer in [25] to estimate velocities using wheel speed sensors, braking torques and longitudinal/lateral accelerometer measurements. Their 
approach utilizes a sliding-mode observer for the velocity estimation and an EKF for estimation of the Burckhardt tire model's friction parameter.

In real situations, a tire model is highly dependent on the presence of tire wear, variable tire parameters, inflation pressure, and uncertainties in road conditions. Therefore, developing an observer for the velocity estimation robust to road conditions and fairly insensitive to tire parameters is desirable. A time-varying Kalman observer is proposed in [26] for longitudinal force estimation using wheel dynamics as well as longitudinal speed estimation at each corner with known and stochastic initial conditions and without road friction information, but utilizes derivatives of the LuGre model's internal states.

This study thus focuses on a method that treats the road condition and acceleration measurement noises as uncertainties. Its observations are also based on tire forces, which are accessible based on wheel dynamics using an unknown input observer [27] or a Kalman-based estimation [28] whenever measured (or estimated) effective torque is available. The proposed velocity estimator in this article uses a parameter-varying observer which can address high-slip conditions in different speed.

This article has been divided into five sections. A longitudinal force estimator is proposed in section II, which includes corner-based force estimation methodology using UKF. Suggested observer and stability analysis of the linear parametervarying (LPV) error dynamics is provided in section III. Section IV contains simulation and experimental results used to verify the approach in various maneuvers and road conditions with high and low longitudinal excitations. Finally, conclusions are provided in section $\mathrm{V}$.

\section{LONGITUDINAL FORCE ESTIMATION}

Longitudinal force estimation significantly contributes to vehicle stability control in the model-based velocity estimator and tire capacity identifier. Estimation of longitudinal forces independent from the road condition may be classified on the basis of wheel dynamics into the Kalman-based estimation [26], [28], [29] and the nonlinear observers [27], [30], [31].

\section{A. Force estimation with the unscented Kalman filter}

The following describes the proposed UKF implementation for longitudinal tire force estimation. Julier et al. [16] proposed a deterministic sampling approach, namely UKF, for state and parameter estimation in discrete-time nonlinear systems and to overcome the linearization problem of the extended Kalman filter. Their method was modified later with augmented states in [32]. Proper capturing of nonlinearities contributed to the unscented transformation that defines the sample vectors $\bar{P} \in \mathbb{R}^{N \times 2 N+1}$ around states where $N$ is the length of the state vector. With some minor changes, UKF can also be employed for the parameter estimation instead of state estimation as provided in [33], [34] for the vehicle parameter identification and in [28] for the longitudinal force estimation. The wheel dynamics at each corner yields:

$$
\bar{T}-R_{e} F_{x}-C_{b} \omega+\Omega_{F}=I_{w} \dot{\omega}
$$

where $R_{e}$ is the wheel effective radius, $F_{x}$ is the longitudinal tire force, $\omega$ is the wheel rotational velocity, $I_{w}$ is the wheel's moment of inertia, $C_{b}$ shows the wheel bearing's linear viscous damping, and $\Omega_{F}$ represents uncertainties in the system including wheel torques, effective radius, and forces. The total effective torque on the wheels is shown by $\bar{T}=T_{t r}-T_{b r}$, whereas traction and braking torques are denoted by $T_{t r}$ and $T_{b r}$ correspondingly. For the proposed UKF-based force estimation, the effective torque $T_{t}$ provides input; the wheel speed $\omega$ is available and assumed to be the measurement $y_{k}$, and the longitudinal force $\hat{F}_{x}$ is treated as the parameter $\hat{p}$. The discrete-time parameter estimation problem then can be expressed as:

$$
\begin{array}{r}
p_{k+1}=p_{k}+\varrho_{k}^{p} \\
y_{k}=\mathcal{G}\left(x_{k}, p_{k}\right)+\varrho_{k}^{m},
\end{array}
$$

where $y_{k}$ corresponds to nonlinear observation on $p_{k}$ and $\varrho_{k}^{p}, \varrho_{k}^{m}$ represent process and measurement noises respectively. The estimated mean is updated as $\hat{p}_{m k}=\hat{p}_{k-1}$ and initialized by $\hat{p}_{0}=\mathbb{E}[p]$. The sample points $\bar{P}_{k \mid k-1}=$

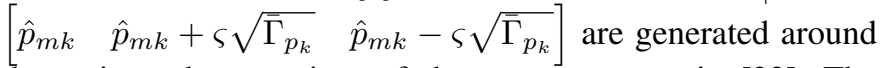
the estimated mean $\hat{p}_{m k}$ of the parameters as in [32]. The square root factorization of the covariance matrix $\bar{\Gamma}_{p_{k}}$ can be obtained by Cholesky decomposition at each time step $k$. The error covariance matrix of the estimated parameter is initialized with $\Gamma_{p_{0}}$ and updated by $\bar{\Gamma}_{p_{k}}=\Gamma_{p_{k-1}}+\rho_{k-1}^{p}$ with incorporation of the process noise covariance $\rho_{k-1}^{p}$. Furthermore, $\varsigma=\sqrt{N+\eta_{1}}$ is a scalar and represents the spread of sample points far from the mean values of random variables, where $\eta_{1}$ is the compound scaling parameter as $\eta_{1}=\epsilon^{2} N-N$ and $\epsilon=\sqrt{3 / N}$. Afterward, $\eta_{2}=2$ is introduced to employ the prior information on the Gaussian distribution of the state/parameter. Generated sample points are supposed to be propagated within the system (wheel dynamics) as the function output $\mathcal{Y}_{k \mid k-1}=\mathcal{G}\left(x_{k}, \bar{P}_{k \mid k-1}\right)$ with the conventional unscented transformation pattern. The output $\hat{y}_{k}$ is achievable from the expected value [35]:

$$
\hat{y}_{k}=\sum_{i=0}^{2 N} W_{i}^{m} \mathcal{Y}_{i, k \mid k-1} .
$$

The weighting coefficients are also defined by $W_{i}^{c}=W_{i}^{m}=$ $\frac{1}{2}\left(N+\eta_{1}\right)$ for all sets $i \in\{1,2, \ldots, 2 N\}$. These coefficients are $W_{0}^{c}=\frac{\eta_{1}}{N+\eta_{1}}+1-\epsilon^{2}+\eta_{2}$ and $W_{0}^{m}=\frac{\eta_{1}}{N+\eta_{1}}$ for $i=0$. The estimated function output $\hat{y}_{k}$ from (3) is then employed in the updated covariance matrices $\Gamma_{y_{k} y_{k}}, \Gamma_{p_{k} y_{k}}$ as follows using the measurement noise covariance $\rho_{k}^{m}$ :

$$
\begin{aligned}
\Gamma_{y_{k} y_{k}} & =\sum_{i=0}^{2 N} W_{i}^{c}\left(\mathcal{Y}_{i, k \mid k-1}-\hat{y}_{k}\right)\left(\mathcal{Y}_{i, k \mid k-1}-\hat{y}_{k}\right)^{T}+\rho_{k}^{m} \\
\Gamma_{p_{k} y_{k}} & =\sum_{i=0}^{2 N} W_{i}^{c}\left(P_{i, k \mid k-1}-\hat{p}_{m k}\right)\left(\mathcal{Y}_{i, k \mid k-1}-\hat{y}_{k}\right)^{T} .
\end{aligned}
$$

Implementing covariance matrices (4), the Kalman gain is defined by $\mathcal{K}_{k}=\Gamma_{p_{k} y_{k}} \Gamma_{y_{k} y_{k}}^{-1}$. Finally, the updated parameter and error covariance are expressed as follows where $\hat{p}_{k}$ is the 
updated parameter estimate and the estimated output is denoted by $\hat{y}_{k}[35]$ :

$$
\begin{aligned}
\Gamma_{p_{k}} & =\bar{\Gamma}_{p_{k}}-\mathcal{K}_{k} \Gamma_{z_{k} z_{k}} \mathcal{K}_{k}^{T} \\
\hat{p}_{k} & =\hat{p}_{m k}+\mathcal{K}_{k}\left(y_{k}-\hat{y}_{k}\right) .
\end{aligned}
$$

The estimated longitudinal force at each corner (tire) is $\hat{F}_{x_{k}}:=$ $\hat{p}_{k}$. The developed method in this article uses different process and measurement error covariance matrices for high wheel acceleration cases to address high-slip conditions at which the level of reliance on process and measurement should change, but the proposed approach in [28] does not consider this change.

The moving sample points through the wheel dynamics (1) reduce the estimation fluctuations as discussed in [28], especially during transient regions, even with the presence of major uncertainties such as the road condition and changes in the effective rolling radius during different maneuvers. A small variation of $R_{e}$ may lead to high fluctuations of estimated forces and consequently velocity estimates in conventional model-based estimation methods. This type of sensitivity does not exist in the proposed force estimation method because of model uncertainties defined in UKF. Highly slippery cases on ice has been a challenge for both nonlinear [27], [31] and Kalman-based [28], [29] longitudinal force estimators; it is addressed in this study by covariance matrices changes in case of high wheel acceleration $\dot{\omega}$.

To evaluate the force estimation method, simulations and road experiments are performed. Simulations using an electrified four independent wheel drive SUV are provided in this section. Results of road experiments are also presented in Section IV. Measured vehicle specifications and related parameters for the simulation and experimental validation are listed in Table I:

TABLE I: Vehicle Spec. for Road Experiments \& Simulation

\begin{tabular}{cccc}
\hline Parameter & Unit & Value & Description \\
\hline$m$ & {$[\mathrm{~kg}]$} & 2275 & Vehicle mass \\
$I_{z}$ & {$\left[\mathrm{~kg} \cdot \mathrm{m}^{2}\right]$} & 4610 & Moment of inertia \\
$I_{w}$ & {$\left[\mathrm{~kg} \cdot \mathrm{m}^{2}\right]$} & 1.7 & Wheel moment of inertia \\
$L_{w b}$ & {$[\mathrm{~m}]$} & 2.85 & Vehicle wheel base \\
$b_{f}\left(b_{r}\right)$ & {$[\mathrm{m}]$} & $1.42(1.43)$ & Front (Rear) axle to CG \\
$R_{e}$ & {$[\mathrm{~m}]$} & 0.34 & Effective radius \\
$h$ & {$[\mathrm{~m}]$} & 0.54 & Roll axis height \\
$\operatorname{Tr}_{f}\left(\operatorname{Tr}_{r}\right)$ & {$[\mathrm{m}]$} & $1.6(1.57)$ & Front (Rear) track width \\
\hline
\end{tabular}

The first set of analysis examines the estimated longitudinal forces by UKF with CarSim for some standard maneuvers. Fig. 1 presents estimated forces compared with longitudinal forces measured at a corner (rear-left wheel) in CarSim. The maneuver is a successive accelerations/brakes on a slippery road with the friction coefficient $\mu=0.3$.

Fig. 1 demonstrates good performance of the suggested method even on such slippery road conditions. The observed fluctuations in the force profile curves exist in the CarSim measured forces and are attributed to the requested acceleration with high magnitude without tire capacity, but the suggested longitudinal force estimator provides smooth results.

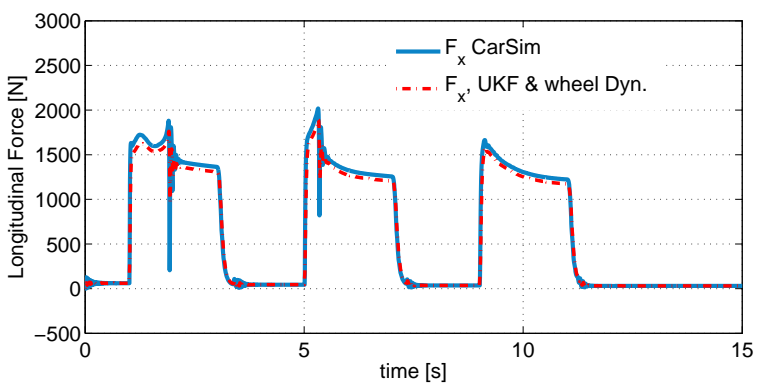

Fig. 1: Estimated $F_{x}$ with UKF on a slippery roads

A standard acceleration-in-turn (AiT) maneuver on dry road is simulated as well, and estimated force results are presented in Fig. 2. Accelerator is applied to $100 \%$ at $t=0.5[s]$ and a $\delta=0.06[\mathrm{rad}]$ steering angle (at tires) is imposed at $t=$ $2[s]$. This maneuver is repeated on a slippery road with $\mu=$ 0.4. Oscillations in the transient regions of this type of harsh maneuver are captured well by the UKF approach.

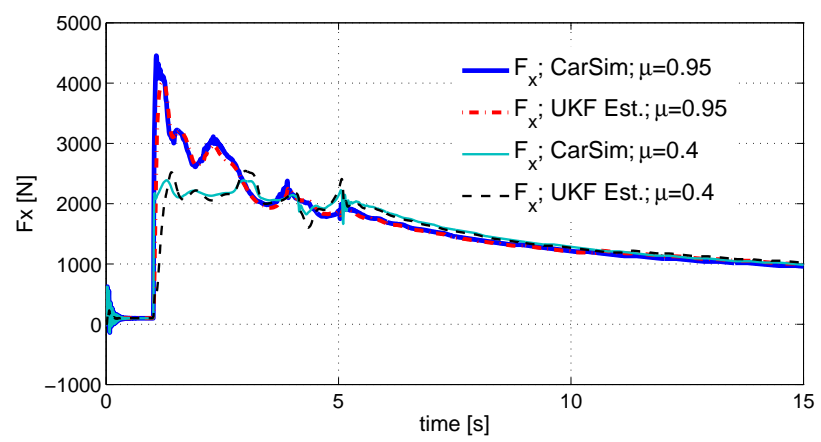

Fig. 2: Long. force Est. for AiT on dry and snowy road

Estimated longitudinal forces are normalized to be utilized in the velocity estimator, which will be explored in the next section. In order to normalize the longitudinal forces, the corner-based normal force estimation approach developed in [26] is employed here in this article. The normalized longitudinal forces are defined as $\mu_{x_{q}}=\hat{F}_{x_{q}} / F_{z_{q}}$ for $q \in$ $\{f L, f R, r L, r R\}$ where $\hat{F}_{x_{q}}$ is the estimated force from (5) and $F_{z_{q}}$ is the normal force from [36] at each tire.

\section{LONGITUDINAL VELOCITY ESTIMATION}

Relying only on the measured acceleration from the stock IMU and using a slip detection algorithm along with a proper stochastic estimator is not sufficient to guarantee the effectiveness of the kinematic-based velocity estimation approaches due to sensor noise (or bias) and uncertainties in the model. Therefore, using vehicle kinematics and accelerations with implementation of an observer on tire forces $\mu_{x_{q}}$ is a reliable approach to estimate a vehicle speed. Although this method seems promising because of the bounded nature of the tire forces, which prohibits accumulated errors, it requires a precise 
tire model, as well as a good perception of the road condition, which does not seem practical.

The key problems of using the tire forces for velocity estimation are in dealing with the road friction and several tire parameters that are time varying. There are at least two approaches to tackle this problem: a) jointly estimating the road condition and slip ratio; b) utilizing an observer-based approach which considers the unknown road condition as a bounded uncertainty, then employing the estimated frictionindependent tire forces for correcting the estimates. In this manuscript, the second method is developed to design an observer at each corner and estimate the longitudinal velocity robust to road conditions. The performance of the proposed method is tested for both dry and slippery roads. The proposed method in this paper has time-varying observer gains with acceptable disturbance attenuation level to tackle very highslip conditions in different speed which is a challenge in [26]. Moreover, the proposed estimator dynamics in [26] uses Gaussian noise assumption and derivatives of the LuGre internal state which should be handled meticulously, but the approach in this article has different estimator dynamics.

The lumped LuGre tire model and its significance on the estimation method are first provided in this section. Then, designing the parameter-varying observer is discussed and its stability and robustness are investigated.

\section{A. The tire model}

The longitudinal/lateral forces generated at each tire's patch during traction, braking, and cornering maneuvers are realized to be dependent on the road condition, slip ratio, slip angle, and normal forces. The steady-state assumption in semi-empirical tire models such as Pacejka, known as "Magic Formula" [37] will not precisely exhibit tire forces during transient acceleration/braking maneuvers. Therefore, dynamic tire models are introduced for considering transient phases, as examined in [19], [38], [39]. Canudas-de-Wit et al. proposed the dynamic LuGre tire-road friction model in [40]-[42] and introduced tire deflection as a state with some simplifications on the normal force distribution by the averaged representation. Velenis et al. provided combined friction characteristics of the average lumped LuGre model in [43]. As a result, longitudinal and lateral tire parameters together with corresponding relative velocities $v_{r i}$ serve as input to the combined slip model:

$$
\dot{\bar{z}}_{i}=v_{r i}-C_{0 i} \bar{z}_{i}-\kappa R_{e}|\omega| \bar{z}_{i}
$$

in which $C_{0 i}=\frac{\left\|M_{c}^{2} \mathbf{v}_{\mathbf{r}}\right\| \sigma_{0 i}}{g\left(\mathbf{v}_{\mathbf{r}}\right) \mu_{c i}^{2}}$ for both longitudinal and lateral directions (i.e., $i \in\{x, y\}), \mathbf{v}_{r}=\left[\begin{array}{ll}v_{r x} & v_{r y}\end{array}\right]^{T}$, and $M_{c}=\left[\begin{array}{lll}\mu_{c x} & 0 ; 0 & \mu_{c y}\end{array}\right]$. The internal state $\overline{\mathbf{z}}=\left[\begin{array}{ll}\bar{z}_{x} & \bar{z}_{y}\end{array}\right]^{T}$ is defined for the patch element at the point $\zeta$ at a time $t$ and the rubber stiffness is denoted by $\sigma_{0}=\left[\begin{array}{lll}\sigma_{0 x} & 0 ; 0 & \sigma_{0 y}\end{array}\right]$. The longitudinal relative velocity is related to the slip ratio $\lambda$ by $v_{r x}=\lambda R_{e} \omega$ and $v_{r x}=\lambda V_{x t}$ for the traction and brake cases, respectively. $V_{x t}$ shows the longitudinal velocity component in the tire coordinates at each corner. Force distribution is represented by parameter $\kappa=\left[\begin{array}{lll}\kappa_{x} & 0 ; 0 & \kappa_{y}\end{array}\right]$ in the average lumped model and could be a constant, or a function of time, or may be approximated by an asymmetric trapezoidal scheme.
The suggested value for $\kappa$ in [42] is $\kappa_{i}=\frac{7}{6 L_{i}}$, where $L$ is the tire patch length. The level of the tire and road adhesion is represented by introducing a so-called "Road Classification Factor", which may vary between $0.1<$ $\theta<0.98$ according to icy, wet, and dry conditions. The transient region between the Columb and static friction in the combined slip tire model is introduced in [44] as $g\left(\mathbf{v}_{\mathbf{r}}\right)=\frac{\left\|M_{c}^{2} \mathbf{v}_{\mathbf{r}}\right\|}{\left\|M_{c} \mathbf{v}_{\mathbf{r}}\right\|}+\left(\frac{\left\|M_{s}^{2} \mathbf{v}_{\mathbf{r}}\right\|}{\left\|M_{s} \mathbf{v}_{\mathbf{r}}\right\|}-\frac{\left\|M_{c}^{2} \mathbf{v}_{\mathbf{r}}\right\|}{\left\|M_{c} \mathbf{v}_{\mathbf{r}}\right\|}\right) e^{-\left|\frac{\left\|\mathbf{v}_{\mathbf{r}}\right\|}{V_{s}}\right|^{\alpha}}$ where $M_{s}=\left[\begin{array}{lll}\mu_{s x} & 0 ; 0 & \mu_{s y}\end{array}\right]$ and $V_{s}$ represents the transition between the Columb and static friction state. The tire parameter $\alpha$ is introduced to shows the steady-state friction and slip interaction.

The final form of the normalized friction force $\boldsymbol{\mu}$ of the average lumped [44] LuGre model yields:

$$
\boldsymbol{\mu}=\sigma_{0} \overline{\mathbf{z}}+\sigma_{1} \dot{\overline{\mathbf{z}}}+\sigma_{2} \mathbf{v}_{r},
$$

where $\boldsymbol{\mu}=\left[\begin{array}{ll}\mu_{x} & \mu_{y}\end{array}\right]^{T}$, the rubber damping is $\sigma_{1}=$ $\left[\begin{array}{lll}\sigma_{1 x} & 0 ; 0 & \sigma_{1 y}\end{array}\right]$, and the relative viscous damping is defined by $\sigma_{2}=\left[\begin{array}{lll}\sigma_{2 x} & 0 ; 0 & \sigma_{2 y}\end{array}\right]$. The vehicle stability is analysed with the pure and combined-slip LuGre tire models in [45]. The steady-state normalized longitudinal LuGre tire forces are shown in Fig. 3 for an acceleration maneuver on roads with different classification numbers $0.3<\theta<0.98$ and longitudinal tire parameters $\sigma_{0 x}=610, \sigma_{2 x}=0.002, \mu_{s}=$ $1.5, \mu_{c}=0.9, V_{s}=6.4, \kappa=8, R_{e}=0.35$, and $\alpha=0.5$.

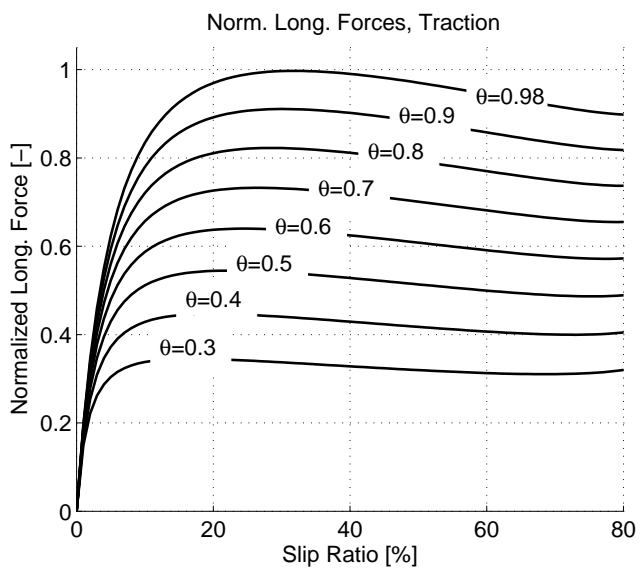

Fig. 3: LuGre tire forces under various road conditions

As can be seen from Fig. 3, the normalized longitudinal tire forces are highly influenced by the road friction even in the linear region. The proposed approach in the following assumes the longitudinal friction term $\frac{\sigma_{0 x}\left|v_{r x}\right|}{\theta g\left(v_{r x}\right)} \bar{z}_{x}$ in (6) as uncertainty and utilizes a linear parameter-varying observer without information on the road friction to estimate the vehicle speed at each tire.

\section{B. State estimation robust to the road condition}

The issues of sensor bias/noise removal in the kinematicbased velocity estimators and unknown model parameters (road friction and tire parameters) in the model-based velocity 
estimators are addressed in the current study using selfbounded tire states. The tire internal deflection state $\left.\bar{z}_{x}(\zeta, t)\right)$ of the average lumped LuGre model has known dynamics and can be used in the velocity estimation as a state. The LuGre internal longitudinal non-measurable deflection states can be written in the presence of uncertainty $\Omega_{1}$ as [46]:

$$
\dot{\bar{z}}_{x}(\zeta, t)=v_{r x}-\kappa_{x} R_{e}|\omega| \bar{z}_{x}(\zeta, t)+\Omega_{1}(t) .
$$

where the longitudinal road friction term $\frac{\sigma_{0 x}\left|v_{r x}\right|}{\theta g\left(v_{r x}\right)} \bar{z}_{x}$ of the LuGre model presented in (6) is replaced with the uncertainty $\Omega_{1}(t)$. This uncertain friction term is amplitude bounded because of the road condition range and physical characteristics of the $g\left(v_{r x}\right)$ function that is defined by $g\left(v_{r x}\right)=\mu_{c x}+$ $\left(\mu_{s x}-\mu_{c x}\right) e^{-\left|\frac{v_{r x}}{V_{s}}\right|^{\alpha}}$. Boundedness of the LuGre model describes that the set $\Delta=\left\{\bar{z}_{x}:\left|\bar{z}_{x}\right| \leq \mu_{s} / \sigma_{0}\right\}$ is an invariant set. Internal state dissipativity (energy-dissipation) shows that the map $v_{r}$ to $\bar{z}_{x}$ is dissipative with respect to the storage function $h\left(\bar{z}_{x}\right)=\bar{z}_{x}^{2} / 2$. Thus, $\int_{0}^{t} v_{r}(\tau) \bar{z}_{x}(\tau) d \tau \geq h\left(\bar{z}_{x}\right)-h\left(\bar{z}_{x}(0)\right)$ for all $t \geq 0$. This characteristic confirms that if all parameters of the model are positive, the model is input to state passive [47] and produce bounded outputs for bounded inputs.

In order to construct a state representation, the derivative of the relative velocity is required. This derivative is also corrupted due to the sensor noise [26]:

$$
\dot{v}_{r x}=R_{e} \dot{\omega}-\dot{V}_{x t}+\Omega_{2}(t),
$$

where the rotational acceleration is $\dot{\omega}$ and $\Omega_{2}(t)$ shows the deviation of the measured relative acceleration $R_{e} \dot{\omega}-\dot{V}_{x t}$ from the system state of $\dot{v}_{r x}$. The mapped longitudinal acceleration of the wheel's center in the tire coordinate system is denoted by $\dot{V}_{x t}$. This mapping is achievable using conventional kinematic equations, vehicle geometry, the yaw rate $r_{C G}$, and both lateral and longitudinal measured acceleration $a_{x_{C G}}, a_{y_{C G}}$, which are corrected by the estimated road angles from an unknown input observer in [48]. The aim is to develop an observer to estimate both the tire deflections $\bar{z}_{x}$ and relative velocities $v_{r x}$ concurrently. The observer output (longitudinal force) is then compared with the one estimated by the UKF (see previous subsection). Consequently, the following observer for the longitudinal velocity estimation is proposed for each corner in which $\mu_{x}$ is the normalized longitudinal force at that corner:

$$
\begin{aligned}
\dot{\bar{z}}_{x}(\zeta, t) & =v_{r x}-\kappa_{x} R_{e}|\omega| \hat{\bar{z}}_{x}(\zeta, t)+L_{1}\left(\mu_{x}-\hat{\mu}_{x}\right) \\
\dot{\hat{v}}_{r x} & =R_{e} \dot{\omega}-\dot{V}_{x t}+L_{2}\left(\mu_{x}-\hat{\mu}_{x}\right) .
\end{aligned}
$$

The output is also the normalized force as:

$$
\hat{\mu}_{x}=\sigma_{0 x} \hat{\bar{z}}_{x}+\sigma_{1 x} \dot{\overline{\bar{z}}}_{x}+\sigma_{2 x} \hat{v}_{r x} .
$$

Therefore, the observer can be written as:

$$
\begin{aligned}
{\left[\begin{array}{c}
\dot{\bar{z}}_{x} \\
\dot{\hat{v}}_{r x}
\end{array}\right] } & =\left[\begin{array}{cc}
-\kappa_{x} R_{e}|\omega| & 1 \\
0 & 0
\end{array}\right]\left[\begin{array}{c}
\hat{\bar{z}}_{x} \\
\hat{v}_{r x}
\end{array}\right]+\left[\begin{array}{c}
0 \\
R_{e} \dot{\omega}-\dot{V}_{x t}
\end{array}\right] \\
& +\left[\begin{array}{c}
L_{1} \\
L_{2}
\end{array}\right]\left(\mu_{x}-\hat{\mu}_{x}\right) \\
& =A(\omega) \hat{x}+B u+L(y-\hat{y}) .
\end{aligned}
$$

Taking into account that the systems dynamic is time-varying with respect to the wheel speed, the suggested estimation method must be designed for the corresponding uncertain linear parameter-varying system. Now, the LPV system could be described as:

$$
\dot{\hat{x}}=A(\omega) \hat{x}+B u+L(y-\hat{y}),
$$

where $\hat{x}(t)=\left[\begin{array}{ll}\hat{\bar{z}}_{x} & \hat{v}_{r x}\end{array}\right]^{T}$; the bounded time-varying parameter is the wheel speed $\omega: \mathbb{R}_{\geq 0} \rightarrow \mathcal{S}_{p}$; and the parameter varying state transition matrix is $A(\omega) \in \mathbb{R}^{2 \times 2}$. The output is the longitudinal normalized force $\hat{\mu}_{x}$, which can be written as:

$$
\hat{y}=C \hat{x}+C^{\prime} \dot{\hat{x}}
$$

with matrices $C=\left[\begin{array}{ll}\sigma_{0 x} & \sigma_{2 x}\end{array}\right]$ and $C^{\prime}=\left[\begin{array}{ll}\sigma_{1 x} & 0\end{array}\right]$. The linear time-varying system presented in (12) and (14) employs a reduced number of tire parameters, such as rubber stiffness $\sigma_{0 x}$, rubber damping $\sigma_{1 x}$, relative viscous damping $\sigma_{2 x}$, and the normal force distribution factor $\kappa$. These tire parameters in the average lumped LuGre model, are not related to the road friction. Thus, the road condition is not required for this tire-based method. If road friction information is available, the estimator would be more accurate and allocated observer gains would be less conservative.

The general structure of the discussed observer is shown in Fig. 4 in which subscript $i j$ represents the estimation at each corner.

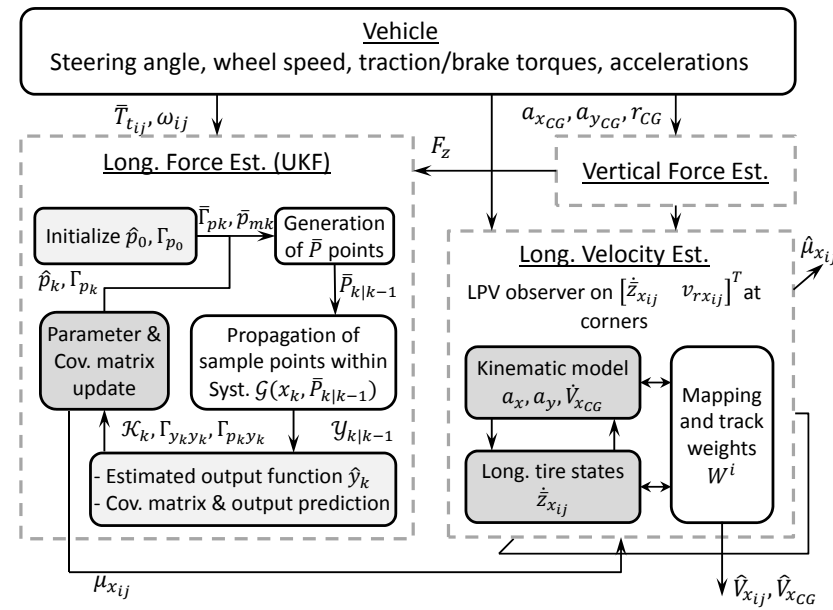

Fig. 4: Structure of the longitudinal velocity estimation

The corner-based Long. Force Est. module provides longitudinal forces using wheel dynamics, the unscented transformation, sample points $\bar{P}_{k \mid k-1}$, wheel torques $\bar{T}_{i j}$, and wheel speeds $\omega_{i j}$. The Long. Velocity Est. module utilizes normalized forces $\mu_{x_{i j}}$, accelerations $a_{x_{C G}}, a_{y_{C G}}$, wheel speed, and yaw rate $r_{C G}$ measurements to estimate relative velocities $v_{r x_{i j}}$ by combining the kinematic approach and the longitudinal tire states $\bar{z}_{x_{i j}}$. The estimated relative velocities at each corner are then mapped to corners and the vehicle's CG to get $\hat{V}_{x_{i j}}, \hat{V}_{x_{C G}}$. The advantage of this structure is that it does not need road friction information. Robustness of this approach to 
disturbances and its Stability are next discussed in this section. Front and rear axle weights (gains) $W^{i}$ are also implemented to have more reliable estimates at vehicle's CG and tires.

Remark 1: The linear system (12) with output $y=C x+$ $C^{\prime} \dot{x}$ is observable. Note that the output equation may be written as $y=\left(C+C^{\prime} A(\omega)\right) x+C^{\prime} B u$. Hence, the observability matrix for this system yields [49]:

$$
\begin{array}{r}
\mathcal{O}_{n}=\left[\begin{array}{lll}
\tau_{1} & \tau_{2} \ldots & \tau_{n}
\end{array}\right]^{T} \\
\tau_{1}=C+C^{\prime} A(\omega), \quad \tau_{i+1}=\tau_{i} A+\dot{\tau}_{i} .
\end{array}
$$

To check the observability condition (15), determinant of $\mathcal{O}_{n}$ is graphically illustrated in Fig. 5. As can be seen from Fig. 5, the observability determinant is always positive for the specified ranges of the wheel speed and its derivative. Thus, the observability matrix $\mathcal{O}_{n}$ has the full rank and the suggested parameter-varying system, (12) is observable.

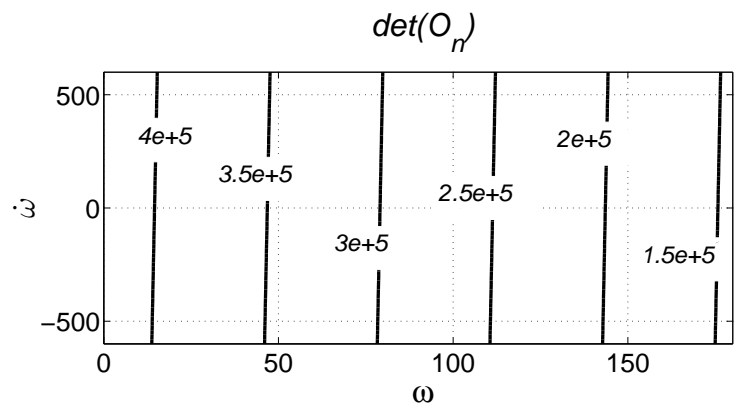

Fig. 5: Observability study for the operating regions

Therefore, it is feasible to observe the longitudinal tire internal states $\bar{z}_{x}$ and the relative velocity $v_{r x}$ by employing the normalized force measurement as the output. The estimation error can be defined as $e=x-\hat{x}$. Thus, the estimation error dynamic is as follows:

$$
\dot{e}(t)=A_{e}(\omega) e(t)+A_{\Omega} \Omega,
$$

where $A_{e}(\omega)=\left(I+L C^{\prime}\right)^{-1}(A(\omega)-L C), A_{\Omega}=(I+$ $\left.L C^{\prime}\right)^{-1}$, and $\Omega \in \mathbb{R}^{2}$ contains uncertainties and is defined by $\left[\begin{array}{ll}\Omega_{1} & \Omega_{2}\end{array}\right]^{T}$.

\section{Stability analysis}

This section first investigates the stability and performance of the constant gain observer obtained based on experiment. Afterward, it is shown that the extension of the current method to the parameter-varying observer design improves the performance of the estimator.

To study the quadratic stability of an affinely dependent system, it is convenient to assume lower and upper bounds for the parameters $\rho_{i} \in\left[\rho_{l i}, \rho_{u i}\right]$ and for the corresponding rate of the parameters $\dot{\rho}_{i} \in\left[\dot{\rho}_{l i}, \dot{\rho}_{u i}\right]$. The set of vertices of the parameter box is denoted by $\mathcal{S}_{p}:=\left\{\left(\nu_{1}, \ldots, \nu_{f}\right) \mid \nu_{i} \in\left[\rho_{l i}, \rho_{u i}\right]\right\}$ and the set of corners of the parameters rate box are also represented by
$\mathcal{S}_{r}:=\left\{\left(\psi_{1}, \ldots, \psi_{f}\right) \mid \psi_{i} \in\left[\dot{\rho}_{l i}, \dot{\rho}_{u i}\right]\right\}$. The following parametervarying system is then assumed with an initial condition of $x(0)=x_{0}$ for all $t \geq 0$.

$$
\dot{x}(t)=\bar{A}(\rho) x(t) .
$$

The state matrix $\bar{A}(\rho)$ is said to be affinely dependent on the parameters $\rho_{i}$ when known and fixed matrices $a_{0}, a_{1}, \ldots, a_{f}$ exist such that $\bar{A}(\rho)=a_{0}+\rho_{1} a_{1}+\ldots+\rho_{f} a_{f}$. The system (17) is defined as robustly stable [50] if the positive definite $V(x, \rho)=x^{T} P(\rho) x$ is a Lyapunov function for such a system and $P(\rho) \succ 0$. Addressing the robust stability of the parametervarying system (17) and dealing with linear matrix inequalities for all trajectories of the parameter $\rho(t)$ is hard in general, thus less conservative approaches, such as affine quadratic stability, to evaluate the robust stability are of more interest.

Theorem 1: [51] The linear system (17) is affinely quadratically stable over all possible trajectories of the parameter vector $\left(\rho_{1}(t), \ldots, \rho_{f}(t)\right)$ if $\bar{A}\left(\rho_{m}\right)$ is stable $\left(\rho_{m}\right.$ is the average of the parameter span) and there exists an affine positive definite Lyapunov function $V(x, \rho)=x^{T} P(\rho) x$ with $P(\rho)=$ $P_{0}+\rho_{1} P_{1}+\ldots+\rho_{f} P_{f} \succ 0$ such that $d V(\nu, \psi) / d t \prec 0$ for all initial conditions $x_{0}$ and the additional multi-convexity constraint $a_{i}^{T} P_{i}+P_{i} a_{i} \succeq 0$ holds for all $i \in\{1, \ldots, f\}$ where $a_{i}$ are fixed matrices in $\bar{A}(\rho)$. The condition $\dot{V}(\nu, \psi) \prec 0$ resembles.

$$
\bar{A}^{T}(\nu) P(\nu)+P(\nu) \bar{A}(\nu)+P(\psi)-P_{0} \prec 0,
$$

for all $(\nu, \psi) \in \mathcal{S}_{p} \times \mathcal{S}_{r}$. The foremost reason for adding the multi-convexity constraint $a_{i}^{T} P_{i}+P_{i} a_{i} \succeq 0$ is that finding $f+1$ symmetric matrices $P_{0}, \ldots, P_{f}$ to make $P(\rho) \succ 0$ and satisfying (18) is itself a nonconvex problem. The affine quadratic stability condition implements the variation rate $\dot{\rho}(t)$, which makes it less conservative than the quadratic stability criteria

Proposition 1: The error dynamics (19) of the suggested observer is affinely quadratically stable with respect to parameter $\omega$ in the sets $\mathcal{S}_{p}=\left[\omega_{l}, \omega_{u}\right], \mathcal{S}_{r}=\left[\dot{\omega}_{l}, \dot{\omega}_{u}\right]$ with constant observer gains of $L=\left[\begin{array}{ll}L_{1} & L_{2}\end{array}\right]$ for all $t \geq 0$ :

$$
\dot{e}(t)=A_{e}(\omega) e(t)
$$

Proof: The stability of the linear time-varying error dynamics can be addressed with rewriting the state matrix $A_{1}(\omega)$ in the form of affinely dependent $A_{e}(\omega)=a_{0}+\omega a_{1}$ where

$$
a_{0}=\left[\begin{array}{cc}
\frac{-L_{1} \sigma_{0}}{1+L_{1} \sigma_{1}} & \frac{1-L_{1} \sigma_{2}}{1+L_{1} \sigma_{1}} \\
\frac{-L_{2} \sigma_{0}}{1+L_{1} \sigma_{1}} & \frac{-L_{2}\left(\sigma_{1}+\sigma_{2}\right)}{1+L_{1} \sigma_{1}}
\end{array}\right] ; a_{1}=\left[\begin{array}{cc}
-\kappa_{x} R_{e} & 0 \\
\frac{L_{2} R_{e} \sigma_{1} \kappa}{1+L_{1} \sigma_{1}} & 0
\end{array}\right] \text {. }
$$

The wheel speed is a bounded variable with operating region of $\omega \in\left[\omega_{l}, \omega_{u}\right]$. The rate of variation is also bounded between $\dot{\omega} \in\left[\dot{\omega}_{l}, \dot{\omega}_{u}\right]$. Now consider the Lyapunov function $V(x)=$ $x^{T}\left(P_{0}+P_{1} \omega\right) x$ for analyzing the system stability. The affine matrix $P(\omega)=P_{0}+\omega_{1} P_{1}$ can be found numerically for each set of observer gains $L_{1}, L_{2}$ such that LMIs (18) holds for all $(\nu, \psi) \in \mathcal{S}_{p} \times \mathcal{S}_{r}$ and $a_{1}^{T} P_{i}+P_{i} a_{1} \succeq 0$.

For the specific case in this article, two observer gains $L_{1}=0.65, L_{2}=197$ are obtained by several simulations and 
experimental tests on different road conditions. Thus, stability of the system (19) will be guaranteed with the substitution of the operating region $|\omega| \leq 180[\mathrm{rad} / \mathrm{s}]$. The rate of variation is also assumed to be $|\dot{\omega}| \leq 600\left[\mathrm{rad} / \mathrm{s}^{2}\right]$, which is practical for this case according to the sampling frequency $200[\mathrm{~Hz}]$ and measurement errors in the wheel speed. Given the tire specifications in the previous section, observer gains, and the affinely dependent form of (20), the numeric values of the symmetric matrix $P(\omega)$ results in

$$
P(\omega)=\left[\begin{array}{cc}
1 e-4 \omega+0.024 & 0.005 \omega-3.54 \\
0.005 \omega-3.54 & 2744-11.43 \omega
\end{array}\right]
$$

\section{Performance analysis}

Consider a linear parameter-varying system (22) with bounds for parameters and their rates and affine state and input matrices $\bar{A}(\rho), \bar{B}(\rho)$ : with uncertainties $\bar{\Omega}$ in (22):

$$
\begin{aligned}
& \dot{x}(t)=\bar{A}(\rho) x(t)+\bar{B}(\rho) \bar{\Omega} \\
& y(t)=\bar{C}(\rho) x(t)+\bar{D}(\rho) \bar{\Omega},
\end{aligned}
$$

where $x \in \mathbb{R}^{n}$, and $y \in \mathbb{R}^{m}$. The robustness performance of such system can be addressed by several methods, such as quadratic stability tests [52], $K_{m}$ or real $\mu$ analysis [53], [54], Integral Quadratic Constraint, IQC [55], and the affine quadratic $\mathcal{H}_{\infty}$ performance analysis [51]. Bounded Real Lemma secures:

$$
\|y\|_{L_{2 \epsilon}}<\gamma\|\bar{\Omega}\|_{L_{2 \epsilon}}
$$

for all $L_{2}$ bounded input $\bar{\Omega}$ where $\gamma$ is the affine quadratic $\mathcal{H}_{\infty}$ performance. Solving set of LMIs, obtained by bounded real lemma inequality, for all possible trajectories of the parameter vector imposes an infinite number of constraints on unknown $P_{i}$. To resolve this issue, Gahinet et al. in [51] imposed a multiconvexity constraint for the such affine system. Thus, a linear system (22) has affine quadratic $\mathcal{H}_{\infty}$ performance $\gamma$ if there exists $f+1$ symmetric matrices $P_{i}$ such that $P(\rho)=P_{0}+$ $\rho_{1} P_{1}+\ldots+\rho_{f} P_{f} \succ 0$ and (24) holds for all $(\nu, \psi) \in \mathcal{S}_{p} \times \mathcal{S}_{r}$ :

$$
\left[\begin{array}{ccc}
\overline{\mathcal{T}}(\nu, \psi) & P(\nu) \bar{B}(\nu) & \bar{C}^{T}(\nu) \\
\bar{B}^{T}(\nu) P(\nu) & -\gamma I & \bar{D}^{T}(\nu) \\
\bar{C}(\nu) & \bar{D}(\nu) & -\gamma I
\end{array}\right] \prec 0,
$$

where $\overline{\mathcal{T}}(\nu, \psi)=\bar{A}^{T}(\nu) P(\nu)+P(\nu) \bar{A}(\nu) P(\psi)-P_{0}$. This suggests solving (24) instead of solving LMIs for infinite allowable trajectories of the parameter vector $\left[\rho_{1}(t), \ldots, \rho_{f}(t)\right]$. The additional multi-convexity constraint should be taken into account for $i=1, \ldots, n$

$$
\left[\begin{array}{cc}
a_{i}^{T} P_{i}+P_{i} a_{i} & P_{i} b_{i} \\
b_{i}^{T} P_{i} & 0
\end{array}\right] \succeq 0 .
$$

The above affine quadratic $\mathcal{H}_{\infty}$ performance method incorporates the rate of parameter variation, which is an advantage of this method as it addresses the conservativeness and infinite

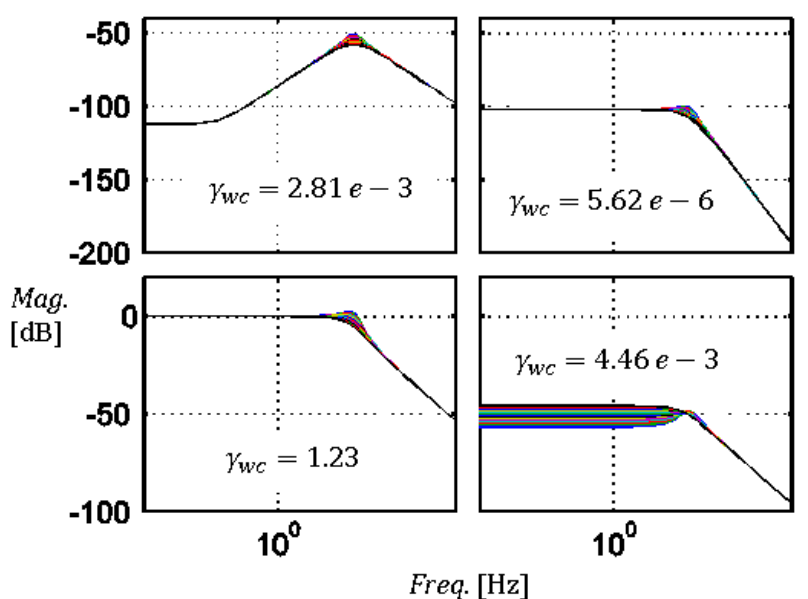

Fig. 6: Response to uncertainties for $L_{1}=0.65, L_{2}=197$

number of constraints induced by the quadratic $\mathcal{H}_{\infty}$ performance. Using observer gains $L_{1}=0.65, L_{2}=197$, the worst case gains $\gamma_{w c}$ of the system are illustrated in Fig. 6.

Figure 6 demonstrates diminishing uncertainties with the allocated gains in all channels except one that shows poor performance $\gamma_{w c}=1.23$ of measurement noise rejection in the second channel of the observer at low frequencies. To resolve this problem, the observer is designed for such LPV system in the following subsection.

\section{E. LPV observer design}

Taking into account that the wheel speed changes significantly in different maneuvers, designing LPV gains for the suggested observer is necessary for capturing the parametervarying characteristics of the wheel speed. The current setting of the observer leads to a nonlinear matrix inequality; thus, a transformation is proposed to linearize the system. This enables us to cast the design procedure in an LMI that can be solved properly. One can choose the following change of variables:

$$
L_{1}=\frac{1-t_{1} \sigma_{1}}{t_{1} \sigma_{1}^{2}} ; L_{2}=\frac{-t_{2}}{t_{1} \sigma_{1}} .
$$

Considering $T=\left[\begin{array}{ll}t_{1} & t_{2}\end{array}\right]^{T}, C_{t}=\left[\begin{array}{ll}\sigma_{0}-\kappa R_{e} \omega \sigma_{1} & \sigma_{1}+\sigma_{2}\end{array}\right]$ and substitution of (26) into (16) yield:

$$
\begin{gathered}
\dot{e}=\left(A_{t}+T C_{t}\right) e+E \Omega \\
y_{e}=\left[\begin{array}{ll}
0 & 1
\end{array}\right]\left[\begin{array}{l}
e_{z_{x}} \\
e_{v_{r x}}
\end{array}\right]=H e,
\end{gathered}
$$

in which $E=T E_{0}+E_{1}, E_{0}=\left[\begin{array}{ll}\sigma_{1} & 0\end{array}\right], E_{1}=\left[\begin{array}{lll}0 & 0 ; 0 & 1\end{array}\right]$ and:

$$
A_{t}=\left[\begin{array}{cc}
\frac{-\sigma_{0}}{\sigma_{1}} & \frac{-\sigma_{2}}{\sigma_{1}} \\
0 & 0
\end{array}\right]
$$

The aim is to find the observer gains such that the ratio of the estimation error to the disturbance energy is minimized 
considering the fact that the process disturbance $\Omega_{1}$ and the measurement disturbance $\Omega_{2}$ are $L_{2 \epsilon}\left[0, t_{f}\right]$ signals. This can be expressed as follows with $\Omega(\theta) \neq 0, e(0)=0$ :

$$
\int_{0}^{t_{f}} y_{e}^{T}(\theta) y_{e}(\theta) d \theta \leq \gamma^{2} \int_{0}^{t_{f}} \Omega^{T}(\theta) \Omega(\theta) d \theta .
$$

Lemma 1: Given a compact set $\omega \in\left[\omega_{l}, \omega_{u}\right]$ and a bounded rate of variation of $|\dot{\omega}|<\psi$, the system (27) is robustly exponentially stable if there exist a continuously differentiable positive definite matrix $P(\omega)$ and a matrix $\eta(\omega)$ such that the following LMI (30) holds. The induced $L_{2 \epsilon}$ norm from the input disturbance to the output error is less than the performance level $\gamma>0$

$$
\left[\begin{array}{ccc}
\mathcal{T}(\omega) & \eta(\omega) E_{0}+P(\omega) E_{1} & H^{T} \\
* & -\gamma I & 0 \\
* & * & -\gamma I
\end{array}\right] \prec 0,
$$

where $P(\omega) T(\omega)=\eta(\omega), \mathcal{T}(\omega)=A_{t}^{T} P(\omega)+P(\omega) A_{t}+$ $C_{t}^{T}(\omega) \eta(\omega)+\eta(\omega) C_{t}(\omega)+\frac{\partial P}{\partial \omega} \dot{\omega}$ and symmetric terms in symmetric matrices are denoted by $*$.

Proof: Consider $V(x, \omega)=x^{T} P(\omega) x$. Taking derivative of the function over the system trajectory yields:

$$
\dot{V}(t)=x^{T}\left[\left(A_{t}^{T}+C_{t}^{T} T^{T}\right) P+P\left(A_{t}+T C_{t}\right)+\frac{\partial P}{\partial \omega} \dot{\omega}\right] x,
$$

which results in:

$$
\begin{aligned}
\left(A_{t}^{T}+C_{t}^{T}(\omega) T^{T}\right) P(\omega)+P(\omega)( & \left.A_{t}+T C_{t}(\omega)\right) \\
& +\frac{\partial P}{\partial \omega} \dot{\omega} \prec 0 .
\end{aligned}
$$

Substituting conditions (32) in the Bounded Real Lemma, one can write the following inequalities:

$$
\left[\begin{array}{ccc}
\mathcal{T}^{\prime}(\omega) & P(\omega)\left(T E_{0}+E_{1}\right) & H^{T} \\
* & -\gamma I & 0 \\
* & * & -\gamma I
\end{array}\right] \prec 0
$$

in which $\mathcal{T}^{\prime}(\omega)=\left(A_{t}^{T}+C_{t}^{T}(\omega) T^{T}\right) P(\omega)+$ $P(\omega)\left(A_{t}+T C_{t}(\omega)\right)+\frac{\partial P}{\partial \omega} \dot{\omega}$. Inequalities (33) guarantees that

$$
\dot{V}+y_{e}^{T} y_{e}-\gamma^{2} \Omega^{T} \Omega<0 .
$$

Taking the integration and considering $V(0)=0, V\left(t_{f}\right)>0$ results in $\int_{0}^{t_{f}} y_{e}^{T}(\theta) y_{e}(\theta) d \theta \leq \gamma^{2} \int_{0}^{t_{f}} \Omega^{T}(\theta) \Omega(\theta) d \theta$ for any nonzero $\omega$.

The final result will be obtained by $T(\omega)=P(\omega)^{-1} \eta(\omega)$. To solve the infinite dimensional parameter-varying LMI (30), one can define the parametric matrices using appropriate basis functions to explicitly turn the LMI into finite dimensional problem. For the sake of simplicity, we defined $P(\omega):=$ $\sum_{i=0}^{f} P_{i} \omega^{i}$ and $\eta(\omega):=\sum_{i=0}^{f} \eta_{i} \omega^{i}$.

To estimate the solution of the infinite dimensional convex optimization, we uniformly grid the set $\omega=\left[\begin{array}{ll}0 & 120\end{array}\right]$ to $N_{g r}=$
120 points $\{\omega\}_{k=1}^{N_{g r}}$. Consequently, time-varying observer gains $L_{1}, L_{2}$ are:

$$
\begin{aligned}
L_{1} & =\frac{1.78 \omega^{2}-50.1 \omega-2.83 e+4}{0.0221 \omega^{2}+154 \omega-2.38 e+4} \\
L_{2} & =\frac{4822\left(2.76 \omega^{2}+150 \omega-7.34 e+4\right)}{0.885 \omega^{2}+6140 \omega-9.51 e+5} .
\end{aligned}
$$

Since $|\dot{\omega}|<\psi$ and it enters linearly in the matrix inequalities, one only needs to check the LMI feasibility at the vertices of the produced convex hull.

The condition for getting the $\mathcal{H}_{\infty}$ performance can be checked graphically by plotting $\|H(j \omega)\|$ for different values of the vehicle wheel speed. The corresponding frequency responses of the LPV system with time-varying observer gains are illustrated in Fig. 7 for different wheel speeds.

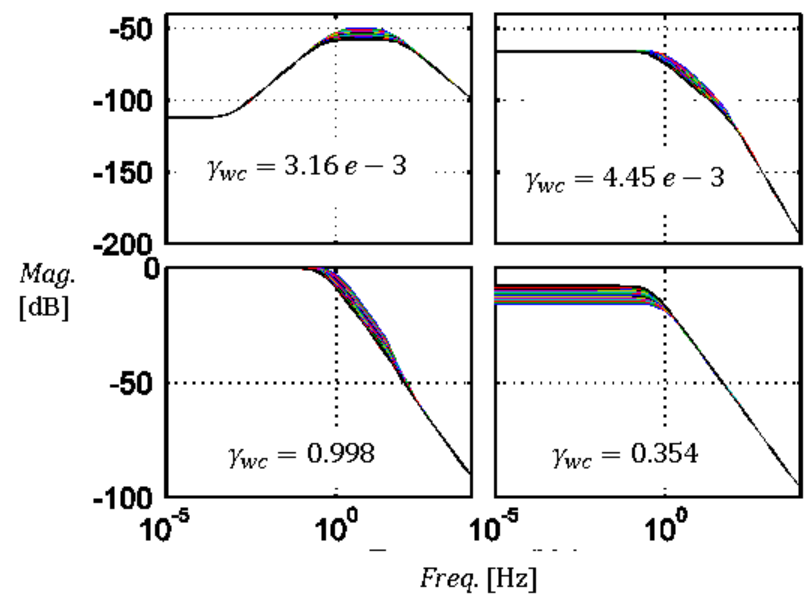

Fig. 7: Response of the observer with time-varying gains

The new observer gains resolve the worst case gain in the low frequency of a channel and provide a more reliable design.

Estimated relative velocities $v_{r x_{i j}}$ at each corner are utilized for $\hat{V}_{x t_{i j}}$ (tires' longitudinal velocities) as $\hat{V}_{x t_{i j}}=R_{e} \omega_{i j}-$ $\hat{v}_{r x_{i j}}$. The estimated velocities in the tire coordinates are then transformed to corners to get the corners' longitudinal velocities $\hat{V}_{x_{i j}}$. Weighted longitudinal velocities at each corner are used for the estimation of the vehicle's CG, $\hat{V}_{x_{C G}}$. Specifically, each axles' longitudinal velocities are defined by $\hat{V}_{x_{f}}$ and $\hat{V}_{x_{r}}$ that are the mean values between $\hat{V}_{x_{f L}}=\hat{V}_{x_{C G}}+r_{C G} \frac{T r_{f}}{2}$ and $\hat{V}_{x_{f R}}=\hat{V}_{x_{C G}}-r_{C G} \frac{T r_{f}}{2}$ for the front axle and $\hat{V}_{x_{r L}}=$ $\hat{V}_{x_{C G}}+r_{C G} \frac{T r_{r}}{2}$ and $\hat{V}_{x_{r R}}=\hat{V}_{x_{C G}}-r_{C G} \frac{T r_{r}}{2}$ for the rear axle, respectively. Then, the velocity of the vehicle's CG is achievable by allocating weights $W^{f}, W^{r}$ to each axle as $\hat{V}_{x_{C G}}=W^{f} \hat{V}_{x_{f}}+W^{r} \hat{V}_{x_{r}}$ with weights $W^{f}=W^{r}=0.5$, but can be allocated based on the slip ratio and the level of reliance on estimated velocities at each track.

\section{RESUlTS AND Discussion}

Several experiments and simulations have been carried out on an instrumented SUV to validate the proposed estimation 
schemes without road friction information. The vehicle is an all-wheel drive (AWD) with an independent electric motor for each wheel. The vehicle specifications are provided in Table I. The vehicle sensors include the steering wheel angle sensor, wheel speed sensors, a 3-axis IMU (and GPS for verification), and sensors to measure each tire's forces and moments (for verification). This test platform is equipped with additional sensors for direct measurement of longitudinal/lateral tire forces at each corner and velocities for evaluation of the force and velocity estimation algorithms. The allocated observer gains in (12) are parameter-varying (with respect to the wheel speed) and obtained from (35) for the friction and acceleration noise effect attenuation respectively.

The followings contain simulation results of the velocity estimation. To check the performance of the estimator during harsh maneuvers, acceleration and brake-in-turn are done and results are illustrated in Fig. 8 and Fig. 9. CarSim simulation results are depicted in Fig. 8 for an acceleration-in-turn (AiT) maneuver. The test is carried out on both dry and slippery $(\mu=0.3)$ roads and the maneuver includes maximum steering angle $\delta=0.05[\mathrm{rad}]$. In addition, the gas pedal is pushed to $100 \%$ for $t=5-10[s]$.

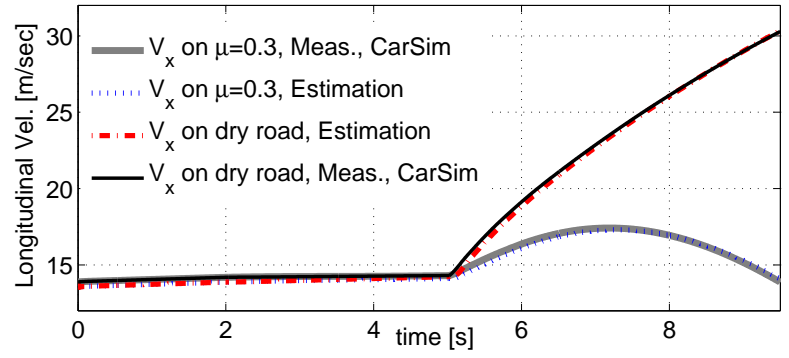

Fig. 8: Estimated $V_{x_{C G}}$, AiT on dry and slippery roads

Velocity estimation is evaluated for a brake-in-turn maneuver (BiT) in Fig. 9, on a slippery road with $\mu=0.25$. The input steering angle $\delta=0.06[\mathrm{rad}]$ is fixed at $t=2[\mathrm{~s}]$ for the entire test, and the brake pedal forces up to $100 \%$ at $t=5[\mathrm{~s}]$.

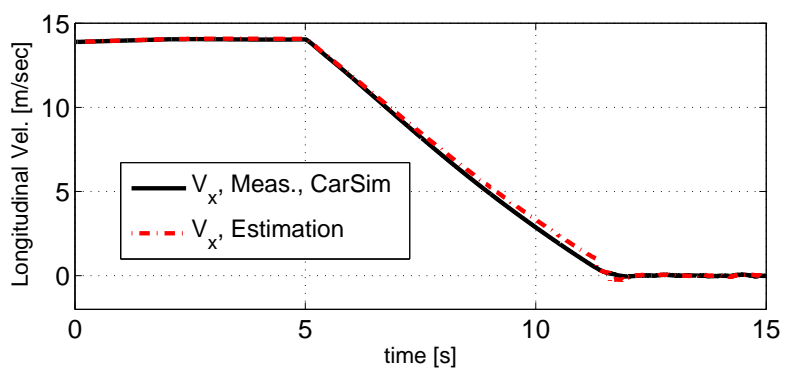

Fig. 9: $V_{x_{C G}}$, Brake-in-turn maneuver, $\mu=0.25$

From the simulation results provided in Fig. 8 and Fig. 9 it is perceptible that the suggested observer can handle both dry and slippery road conditions.
The followings include experimental results on the AWD test platform SUV shown in Fig. 10. Different tests on dry roads, snowy roads, and on test facility surfaces with known friction were executed to validate both the velocity and force estimators.

Measured signals are communicated using a CAN-bus that is a conventional communication method in production vehicles. Real-time logging of this sensory information was done using the dSPACE MicroAutobox shown in Fig. 10. Compiling measurements for MATLAB/SIMULINK were done with the dSPACE, and the controller provides control signals for the actuators through dSPACE. The AUTOBOX has interfaces with actuators (electric motors) and provides control signals.
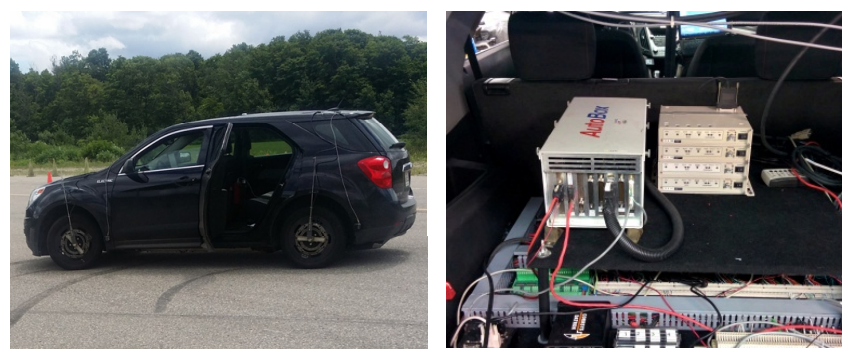

Fig. 10: 4WD SUV test platform and I/O layout

The developed longitudinal force estimator is examined on a wet road with traction and braking and results at the front-right corner tire are compared in Fig. 11-a with measurements and that of the unknown input observer (UIO) method suggested in [27]. Force transducers are mounted on the wheel hub at each corner and are capable of measuring tire forces and moments. The sensors are strain gauge-based rims. The test is executed on a slippery surface with $\mu \approx 0.3$ and the traction configuration is all-wheel drive (AWD). In addition, experimental results of the estimated forces by UIO and UKF are compared with the filtered forces of the wheel hub sensor measured on the rear-left wheel in Fig. 11-b. This maneuver is a double lane change (DLC) on snow with $\mu \approx 0.35$.

A low-pass filter with time constant $\tau=0.04$ is used for removing high oscillations from the measured longitudinal forces. Deviations of the filtered measurements from the estimated forces (by UIO and UKF) in Fig. 11-b are attributed to this filtering for such a harsh maneuver on snow with several steering attempts. These figures show consistency between the outcomes of two methods, but oscillations of the suggested UKF estimator is less than the unknown input observer. The advantage of the proposed force estimation approach over the existing and widely practiced unknown input observers is its reliability; this is due to the stochastic nature of the roadwheel forces, wheel torques, and effective radius, which are considered as uncertainty and has been addressed with the UKF. Moreover, the UKF force estimator can address nonGaussian process or measurement noises.

The proposed velocity estimator is examined on the test vehicle and the following paragraphs provide experimental results. The velocity estimates are employed for traction 

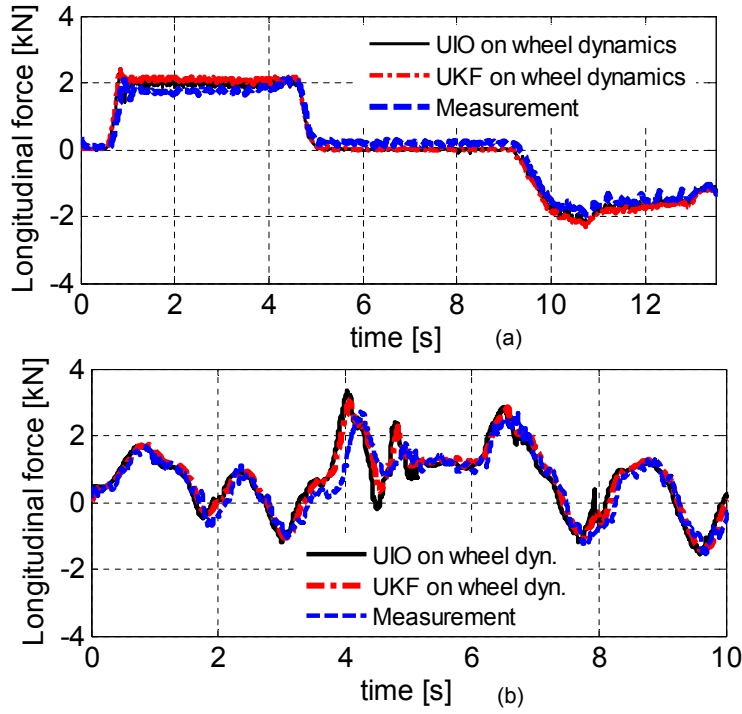

Fig. 11: Longitudinal force Est. (a) Accel./brake on wet (b) DLC on snow

and stability control systems including torque vectoring and differential breaking. The estimator is tested together with the controller in the loop with sampling frequencies of 100 and 200 [Hz]. Fig. 12 depicts estimated longitudinal velocity at vehicle's CG on a dry road with a successive acceleration/deceleration for the AWD case. The GPS is RT2500 and it has slip-angle measurement accuracy of $0.2^{\circ}$ at $50[\mathrm{kph}]$ and longitudinal speed measurement accuracy of $0.1[\mathrm{kph}]$.

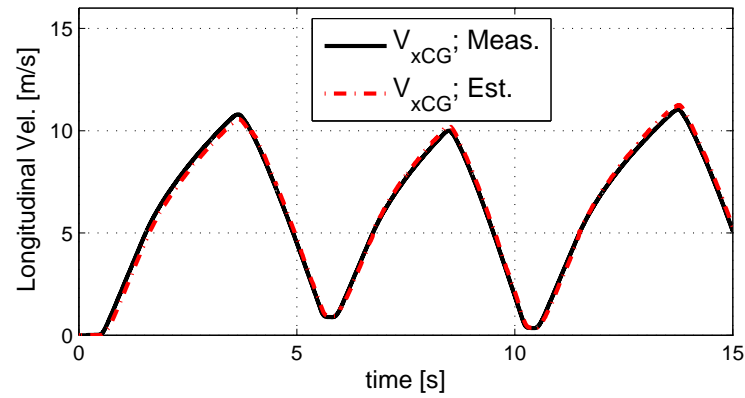

Fig. 12: Long. velocity estimation for Accel./Decel. on dry

One of main objectives of the velocity estimator is to provide reliable $\hat{V}_{x t_{i j}}$ at each corner for the traction/slip control systems during launch/brake on slippery roads. Thus, a launch maneuver on ice in the test facility (with known $\mu \approx 0.2$ ) without any traction control is performed and results are shown in Fig. 13. The developed velocity estimator provides $\hat{V}_{x}$ at CG without road condition information as illustrated in Fig. 13-a; the wheels speed at each corner are shown in Fig. 13-b, which confirms the extreme slip characteristic of this maneuver. There is an almost $60[\mathrm{mSec}]$ delay for the longitudinal speed estimator which is due to the UKF observer for the longitudinal force estimation and the filtered accelerations for the LPV velocity observer.
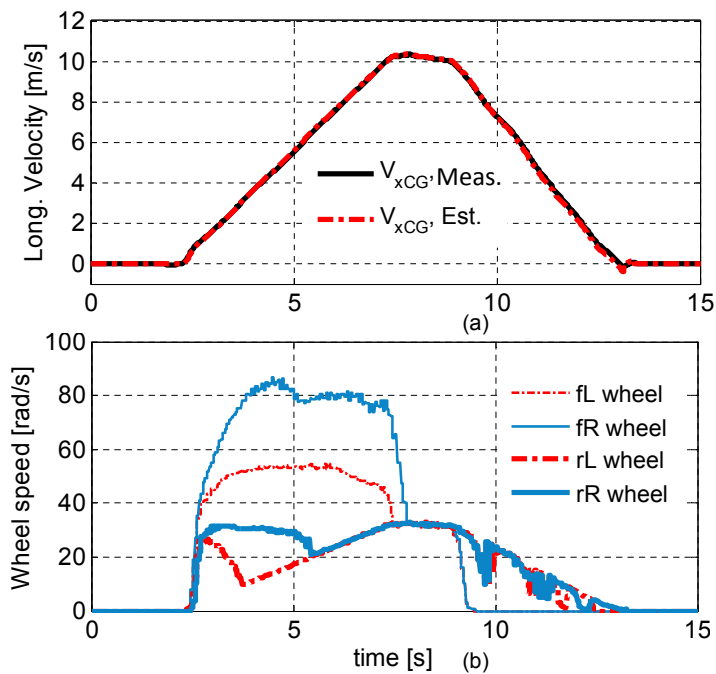

Fig. 13: Launch on ice (a) estimated $\hat{V}_{x_{C G}}$ (b) wheels speed at all corners

The velocity estimator is also studied for combined-slip maneuvers with simultaneous lateral and longitudinal slips and the following tests show the outcomes of the observer for such driving scenarios. A step-steer (SS) maneuver with AWD traction configuration is performed on a wet surface in the test facility with $\mu=0.4$ and the estimated longitudinal velocity is depicted in Fig. 14-a. Although, this maneuver includes transition from wet to dry roads, the estimator provides smooth and reliable results especially for the transient region.
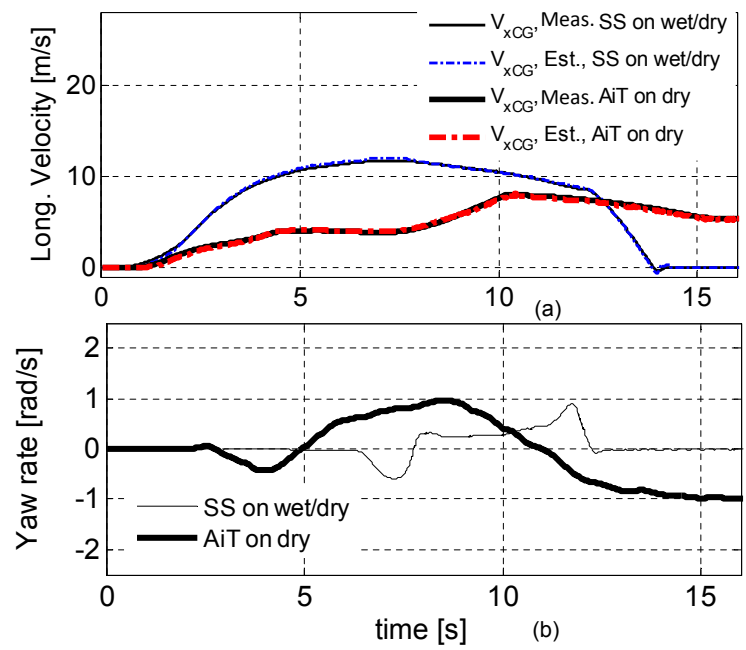

Fig. 14: Acceleration-in-turn and step steer on dry/wet for AWD (a) estimated speed at CG (b) measured yaw rate

In addition, results of the longitudinal velocity estimation 
for an acceleration-in-turn (AiT) scenario on dry asphalt are depicted in Fig. 14-a as well. Difficulties in the maneuvers are represented by changes of the vehicles yaw rate, as shown in Fig. 14-b.

Figure 15 compares experimental results of the newly developed speed estimator for the AWD vehicle with the measured GPS signal on snow. It depicts performance of the approach on slippery roads in maneuvers with combined-slip characteristics such as brake-in-turn (BiT). Furthermore, a lane-change (LC) on a snowy surface with $\mu \approx 0.3$ is performed and the estimation results are depicted in Fig. 15-a.
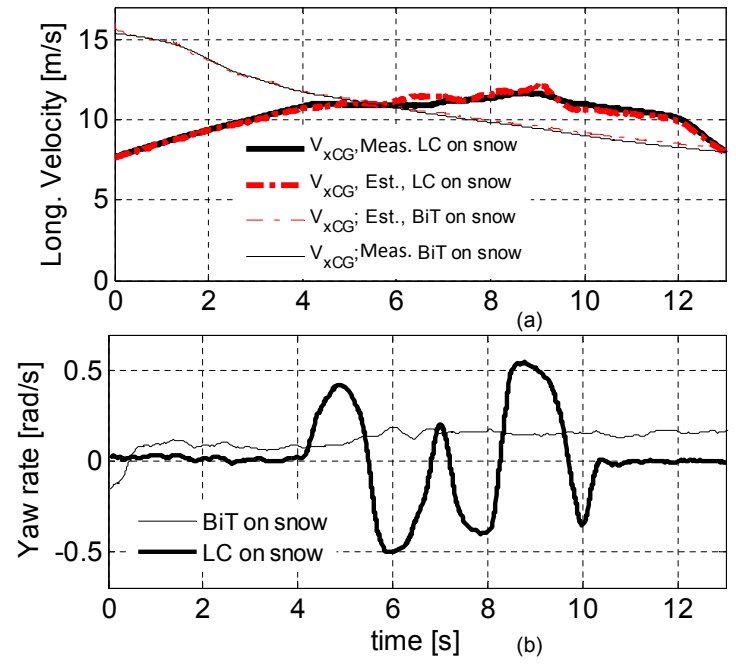

Fig. 15: Brake-in-turn and lane-change on snow/ice

Different traction configurations (all-wheel, front-wheel, and rear-wheel drive) are also allocated to evaluate the cornerbased velocity estimator. For the FWD case, the results are shown in Fig. 16. A launch on a highly slippery wet sealer (with $\mu \approx 0.25$ ) and transition to dry with steering is performed in this maneuver. Front wheels speed in Fig. 16-c confirms high slip ratio due to the low capacity of tires and the load transfer, but the estimated longitudinal velocity exhibits good accuracy.

Performance of the developed Kalman-based estimator in [26] is also compared with the newly proposed LPV observer for the launch on wet sealer in the same figure. As can be seen from Fig. 16, the LPV observer exhibits more accurate estimates specially during large slip incidents. The Kalmanbased approach changes system covariance matrices based on the slip condition and an adaptive weighted axle algorithm to cope with high slip cases, but the LPV method allocates appropriate gains (35) implementing the observer design method discussed in section III.

Velocity estimates of the developed LPV observer at vehicle's CG for the RWD configuration in a launch and deceleration maneuver on the gravel are compared with measurements and Kalman-based longitudinal velocity estimator [26] in Fig. 17-a. Although both methods, show good performance, the LPV approach has better outcomes specially for high

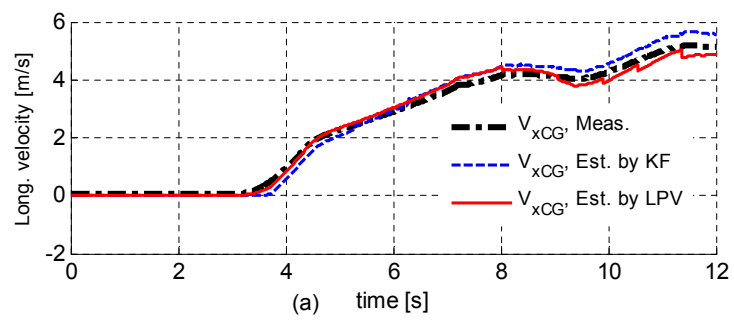

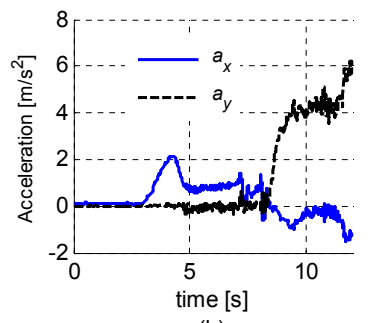

(b)

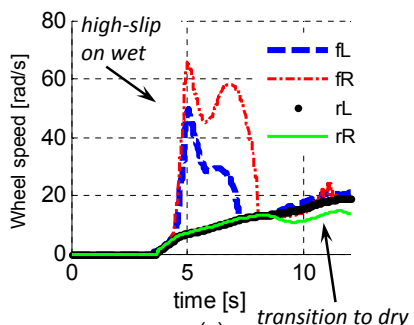

(c)
Fig. 16: Launch on wet asphalt and transition to dry with steering, FWD case (a) $\hat{V}_{x_{C G}}$ (b) accelerations (c) wheel speeds

slip ratio cases on the gravel. The new approach allocates flexible observer gains by solving LMIs (30) with respect to the wheel speed that leads to more accurate velocity estimates. The gravel road has some disturbances on the measured longitudinal/lateral accelerations as depicted in Fig. 17-b. The measured wheel speeds at each corner confirms increase in the slip ratio, but the observer can handle the situation and provides smooth outcomes.
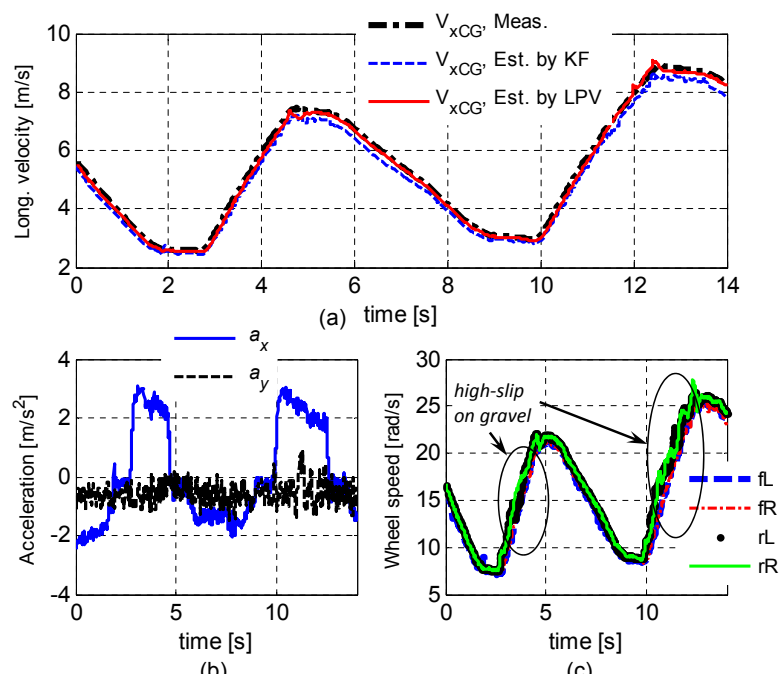

(c)

Fig. 17: Launch and deceleration on gravel, RWD case (a) estimated velocity (b) accelerations (c) wheel speeds

Performance of the longitudinal force and velocity estimators are investigated in Table II using the normalized root mean square of the error, NRMS, for vehicle with $A W D$ and $R W D$ traction configurations in different driving scenar- 
ios. The normalized error root mean square is defined as $\xi_{n}=\frac{\sqrt{\sum_{j=1}^{N_{h}}\left(\hat{h}_{j}-h_{j}^{m}\right)^{2} / N_{h}}}{h}$, where the estimated and measured signals are denoted by $h^{m}$ and $\hat{h}$ respectively, $N_{h}$ is the number of signal samples during a driving scenario, and $\bar{h}=\max _{i=1 \ldots N_{h}}\left|h_{j}^{m}\right|$ shows the maximum value of the measured signal.

TABLE II: Longitudinal state estimators error NRMS

\begin{tabular}{l|ccccc}
\hline \multirow{2}{*}{ Maneuvers } & Est. $V_{x}$ & \multicolumn{4}{c}{ Estimated $F_{x}$} \\
\cline { 2 - 6 } & $\xi_{n}[\%]$ & $\xi_{n 1}$ & $\xi_{n 2}$ & $\xi_{n 3}$ & $\xi_{n 4}$ \\
\hline \hline $\boldsymbol{R W D}$ & 3.44 & 5.18 & 5.3 & 3.25 & 4.87 \\
Launch on ice & 4.65 & 5.71 & 5.56 & 2.63 & 3.22 \\
Launch and brake on gravel & 2.04 & 3.24 & 2.96 & 2.85 & 2.16 \\
DLC on snow & 2.76 & 3.81 & 5.66 & 3.2 & 4.95 \\
\hline $\boldsymbol{A W D}$ & 4.12 & 5.52 & 5.06 & 4.73 & 4.91 \\
Split- $\mu$ launch on dry/ice & 4.85 & 6.42 & 4.13 & 5.41 & 3.78 \\
BiT and LC on snow & & & & & \\
AiT on dry & & & & & \\
\hline
\end{tabular}

The normalized error $R M S$ for the front-left, front-right, rear-left, and rear-right tires are denoted by $\xi_{n 1}, \xi_{n 2}, \xi_{n 3}$, and $\xi_{n 4}$ respectively. With incorporating the tire internal deflection state and time-varying observer gains, the system dynamics suggested in (12) prohibits the states (tire deflections and relative velocities) from high fluctuations and increases accuracy, as is apparent from experimental data. Therefore, the proposed corner-based velocity estimator performs well on dry, wet, and highly slippery roads to provide required measurements for the traction and stability control systems.

\section{CONCLUSION}

This article has proposed and tested experimentally a vehicle state estimation approach. Two main developments have been adopted into the longitudinal state estimation: a) augmented states/parameters in the UKF used for the force estimation; b) velocity estimation with an LPV observer design by decreased sensitivity to the tire parameters and road uncertainties. The uncertainties are mainly rooted in the unknown road friction and the tire parameters. The estimated velocities at each corner and vehicle's CG by the proposed approach are used for the vehicle traction control as well as stability control by differential braking on the test platform. Based on the simulation and experimental results, the following conclusions can be made:

Limitations of the available kinematic-based and model-based estimators lead to an observer scheme robust to road friction changes to estimate the longitudinal velocity at each tire. An LPV observer was designed and variable observer gains are provided with respect to the wheel speed. A method was suggested for analyzing of the affine quadratic stability of the observer's error dynamics. $\mathcal{H}_{\infty}$ performance of the estimator was also investigated with a transformation from nonlinear to linear matrix inequalities.

One significant advantage of the suggested observer is that a unidirectional lumped LuGre model could be used instead of the combined one since the term containing the combined friction model was considered as uncertainty. Finding optimal observer gains that consider both the stability and disturbance rejection properties and rolling resistance effect for longitudinal force estimation will be addressed in the future.

\section{ACKNOWLEDGMENT}

This work was supported by Automotive Partnership Canada, Ontario Research Fund, and the General Motors Co. [grant numbers APCPJ 395996-09 and ORF-RE-04-039].

\section{REFERENCES}

[1] J. Ryu, "State and Parameter Estimation for Vehicle Dynamics Control using GPS," Ph.D. dissertation, Stanford University, 2004.

[2] D. M. Bevly, "Global positioning system (gps): A low-cost velocity sensor for correcting inertial sensor errors on ground vehicles," Journal of dynamic systems, measurement, and control, vol. 126, no. 2, pp. 255-264, 2004.

[3] D. M. Bevly, J. Ryu, and J. C. Gerdes, "Integrating ins sensors with gps measurements for continuous estimation of vehicle sideslip, roll, and tire cornering stiffness," IEEE Transactions on Intelligent Transportation Systems, vol. 7, no. 4, pp. 483-493, 2006.

[4] J. Ryu and J. C. Gerdes, "Integrating inertial sensors with global positioning system (GPS) for vehicle dynamics control," Journal of Dynamic Systems, Measurement, and Control, vol. 126, no. 2, pp. 243254, 2004.

[5] K. Kobayashi, K. Watanabe, and C. Science, "Estimation of absolute vehicle speed using fuzzy logic rule-based Kalman filter," in American Control Conference, 1995, pp. 3086-3090.

[6] Y. Fukada, "Slip-angle estimation for vehicle stability control," Vehicle System Dynamics, vol. 32, no. 4, pp. 375-388, 1999.

[7] A. Hac and M. D. Simpson, "Estimation of vehicle side slip angle and yaw rate," in SAE Technical Paper 2000-01-0696, 2000.

[8] M. Hiemer, A. von Vietinghoff, U. Kiencke, and T. Matsunaga, "Determination of the vehicle body side slip angle with non-linear observer strategies," in SAE Technical Paper 2005-01-0400, 2005.

[9] L. Imsland, T. Johansen, T. Fossen, J. Kalkkuhl, and A. Suissa, "Vehicle velocity estimation using modular nonlinear observers," in Proceedings of the 44th IEEE Conference on Decision and Control, 2005, pp. 67286733.

[10] M. Satria and M. Best, "Comparison between Kalman filter and robust filter for vehicle handling dynamics state estimation," in SAE Technical Paper 2002-01-1185, 2002.

[11] T. a. Wenzel, K. J. Burnham, M. V. Blundell, and R. a. Williams, "Dual extended Kalman filter for vehicle state and parameter estimation," Vehicle System Dynamics, vol. 44, no. 2, pp. 153-171, 2006.

[12] G. Baffet, A. Charara, and G. Dherbomez, "An observer of tireroad forces and friction for active security vehicle systems," vol. 12, no. 6 , pp. 651-661, 2007.

[13] L. Tong, "An approach for vehicle state estimation using extended Kalman filter," in Syst. Simul. Sci. Comput., vol. 1, 2012, pp. 56-63.

[14] M. Burckhardt, Fahrwerktechnik: Radschlupfregelsysteme, Germany, 1993.

[15] X. Huang and J. Wang, "Robust sideslip angle estimation for lightweight vehicles using smooth variable structure filter," in ASME Dynamic Systems and Control Conference, 2013, pp. 1-8.

[16] S. Julier and J. Uhlmann, "A new extension of the Kalman filter to nonlinear systems," in The 11th International Symposium on Aerospace/Defense Sensing, Simulation and Controls, 1997.

[17] E. A. Wan and R. van der Merwe, "The unscented Kalman filter for nonlinear estimation," in Adaptive Sy. for Signal Processing, Соттиnications, and Control Symp., 2000, pp. 153-158.

[18] S. Antonov, A. Fehn, and A. Kugi, "Unscented Kalman filter for vehicle state estimation," Vehicle System Dynamics, vol. 49, no. 9, pp. 14971520, 2011. 
[19] H. B. Pacejka and I. J. M. Besselink, "Magic formula tyre model with transient properties," Vehicle System Dynamics, vol. 27, pp. 234-249, 1997.

[20] M. Wielitzka, M. Dagen, and T. Ortmaier, "State estimation of vehicle's lateral dynamics using unscented kalman filter," in Decision and Control (CDC), 2014 IEEE 53rd Annual Conference on. IEEE, 2014, pp. 50155020 .

[21] C. Lex, A. Eichberger, and W. Hirschberg, "Comprehensive evaluation of different approaches to estimate the tire-road grip potential and their application in driver assistance systems," Proceedings of Chassis Tech, 2011.

[22] G. Magallan, C. H. D. Angelo, and G. O. García, "Maximization of the traction forces in a 2WD electric vehicle," IEEE TRANSACTIONS ON VEHICULAR TECHNOLOGY, vol. 60, no. 2, pp. 369-380, 2011.

[23] B. Li, H. Du, W. Li, and Y. Zhang, "Side-slip angle estimation based lateral dynamics control for omni-directional vehicles with optimal steering angle and traction/brake torque distribution," Mechatronics, vol. 30, pp. 348-362, 2015.

[24] H. Zhang, X. Huang, J. Wang, and H. R. Karimi, "Robust energy-topeak sideslip angle estimation with applications to ground vehicles," Mechatronics, vol. 30, pp. 338-347, 2015.

[25] X. Zhang, Y. Xu, M. Pan, and F. Ren, "A vehicle ABS adaptive slidingmode control algorithm based on the vehicle velocity estimation and tyre/road friction coefficient estimations," Vehicle System Dynamics, vol. 52, no. 4, pp. 475-503, 2014.

[26] E. Hashemi, A. Kasaiezadeh, S. Khosravani, A. Khajepour, N. Moshchuk, and S.-K. Chen, "Estimation of longitudinal speed robust to road conditions for ground vehicles," Vehicle System Dynamics, vol. 54, no. 8, pp. 1120-1146, 2016.

[27] Y. Wang, D. M. Bevly, and S.-k. Chen, "Longitudinal tire force estimation with unknown input observer," in ASME DSCC2012, 2012.

[28] E. Hashemi, A. Kasaeizadeh, A. Khajepour, N. Mushchuk, and S.-K. Chen, "Robust estimation and experimental evaluation of longitudinal friction forces in ground vehicles," in ASME IMECE2014, 2014.

[29] M. Doumiati, A. C. Victorino, A. Charara, and D. Lechner, "Onboard real-time estimation of vehicle lateral tire-road forces and sideslip angle," Mechatronics, IEEE/ASME Transactions on, vol. 16, no. 4, pp. 601-614, 2011.

[30] Y.-H. J. Hsu, S. M. Laws, and J. C. Gerdes, "Estimation of tire slip angle and friction limits using steering torque," Control Systems Technology, IEEE Transactions on, vol. 18, no. 4, pp. 896-907, 2010.

[31] R. Rajamani, G. Phanomchoeng, D. Piyabongkarn, and J. Y. Lew, "Algorithms for real-time estimation of individual wheel tire-road friction coefficients," Mechatronics, IEEE/ASME Transactions on, vol. 17, no. 6, pp. 1183-1195, 2012.

[32] R. V. D. Merwe and E. Wan, "Efficient derivative-free Kalman filters for online learning." in European Symposium on Artificial Neural Networks, ESANN, 2001, pp. 205-210.

[33] S. Hong, T. Smith, F. Borrelli, and J. K. Hedrick, "Vehicle inertial parameter identification using extended and unscented kalman filters," in Intelligent Transportation Systems-(ITSC), 2013 16th International IEEE Conference on. IEEE, 2013, pp. 1436-1441.

[34] S. Hong, C. Lee, F. Borrelli, and J. K. Hedrick, "A novel approach for vehicle inertial parameter identification using a dual kalman filter," Intelligent Transportation Systems, IEEE Transactions on, vol. 16, no. 1, pp. 151-161, 2015.

[35] E. Haykin, Simon, Kalman Filtering and Neural Networks. John Wiley \& Sons, Inc., 2004, vol. 47.

[36] E. Hashemi, M. Pirani, A. Khajepour, B. Fidan, A. Kasaiezadeh, S. Chen, and B. Litkouhi, "Integrated estimation structure for the tire friction forces in ground vehicles," in Advanced Intelligent Mechatronics, 2016 IEEE Conference on. IEEE, 2016.

[37] W. R. Pasterkamp and H. B. Pacejka, "The Tyre as a Sensor to Estimate Friction," Vehicle System Dynamics, vol. 27, pp. 409-422, 1997.
[38] J. Deur, J. Asgari, and D. Hrovat, “A 3D brush-type dynamic tire friction model,” Vehicle System Dynamics, vol. 42, no. 3, pp. 133-173, 2004.

[39] W. Liang, J. Medanic, and R. Ruhl, "Analytical dynamic tire model," Vehicle System Dynamics, vol. 46, no. 3, pp. 197-227, 2008.

[40] C. C. D. Wit, H. Olsson, K. J. Astrom, and P. Lischinsky, "A new model for control of systems with friction," IEEE Transactions on Automatic Control, vol. 40, 1995.

[41] C. Canudas-De-Wit and P. Tsiotras, "Dynamic tire friction models for vehicle traction control," in proceedings of the 38th Conference on Decision \& Control, 1999, pp. 3746-3751.

[42] C. Canudas-de Wit, P. Tsiotras, E. Velenis, M. B. Gissinger, and G. Gissinger, "Dynamic friction models for road/tire longitudinal interaction," Vehicle System Dynamics, vol. 39, pp. 189-226, 2003.

[43] E. Velenis, "Extension of the LuGre dynamic tire friction model to 2D motion," in Proceedings of the 10th IEEE Mediterranean Conference on Control and Automation, 2002, pp. 3746-3751.

[44] E. Velenis, P. Tsiotras, C. Canudas-de Wit, and M. Sorine, "Dynamic tyre friction models for combined longitudinal and lateral vehicle motion," Vehicle System Dynamics, vol. 43, no. 1, pp. 3-29, 2005.

[45] E. Hashemi, M. Pirani, A. Khajepour, and A. Kasaiezadeh, "A comprehensive study on the stability analysis of vehicle dynamics with pure/combined-slip tyre models," Vehicle system dynamics, vol. 54, no. 12, pp. 1736-1761, 2016.

[46] Y. Wang and D. Bevly, "Longitudinal vehicle state estimation; General Motors summary report," General Motors Summary Report, Tech. Rep., 2010.

[47] K. J. Astrom and C. Canudas-de Wit, "Revisiting the LuGre model," IEEE Control Systems Magazine, vol. 6, pp. 101-114, 2008.

[48] E. Hashemi, R. Zarringhalam, A. Khajepour, W. Melek, A. Kasaiezadeh, and S.-K. Chen, "Real-time estimation of the road bank and grade angles with unknown input observers," Vehicle System Dynamics, pp. 1-20, 2017.

[49] R. Tóth, Modeling and Identification of Linear Parameter-Varying Systems. Springer, 2010, vol. 403.

[50] C. Briat, Linear Parameter-varying and Time-delay Systems: Analysis, Observation, Filtering \& Control. Springer, 2015.

[51] P. Gahinet, P. Apkarian, and M. Chilali, "Affine parameter-dependent Lyapunov functions and real parametric uncertainty," IEEE Transactions on Automatic Control, vol. 41, no. 3, pp. 436-442, 1996.

[52] S. Boyd and Q. Yang, "Structured and simultaneous Lyapunov functions for system stability problems," International Journal of Control, vol. 49, no. 6, pp. 2215-2240, 1989.

[53] M. K. H. Fan, A. L. Tits, and J. C. Doyle, "Robustness in the presence of mixed parametric uncertainty and unmodeled dynamics," IEEE Transactions on Automatic Control, vol. 36, pp. 25-38, 1991.

[54] M. Safonov and R. Chiang, "Real/complex k m-synthesis without curve fitting," Control and Dynamic Systems, vol. 56, pp. 303-303, 1993.

[55] H. Pfifer and P. Seiler, "Robustness analysis of linear parameter varying systems using integral quadratic constraints," International Journal of Robust and Nonlinear Control, vol. 25, no. 15, pp. 2843-2864, 2015. 


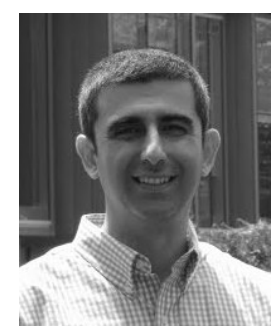

ical systems.
Ehsan Hashemi is currently working toward the $\mathrm{PhD}$ degree in the Department of Mechanical and Mechatronics Engineering, University of Waterloo, Waterloo, ON, Canada. He received his M.Sc. in Mechanical Engineering from Amirkabir University of Technology (Tehran Polytechnic), Tehran, Iran. His previous work includes biped locomotion, control and state estimation of mobile robots, and multibody dynamics. His research interests are fault-toleran control and estimation, robust control, distributed estimation, vehicle active safety systems, and dynam-

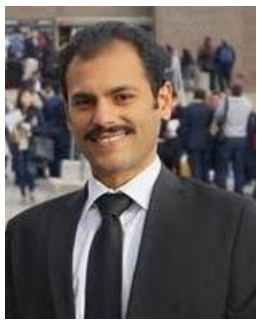

Saeid Khosravani is currently a in the Department of Mechanical and Mechatronics Engineering, University of Waterloo, Waterloo, ON, Canada. He received his M.Sc. in Electrical Engineering in 2011 from Amirkabir University of Technology (Tehran Polytechnic), Tehran, Iran and the Ph.D. degree in mechanical and mechatronics engineering in 2016 from University of Waterloo. His research interests include robust control, vehicle dynamics, large scale systems, fault tolerant control, delay systems, and human in the loop modeling.

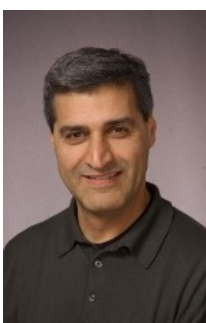

Amir Khajepour is a Professor of mechanical and mechatronics engineering with the University of Waterloo, Waterloo, ON, Canada, and the Canada Research Chair in Mechatronic Vehicle Systems. He has developed an extensive research program that applies his expertise in several key multidisciplinary areas. His research interests include system modeling and control of dynamic systems. His research has resulted in several patents and technology transfers. $\mathrm{He}$ is the author of more than 350 journal and conference publications and five books. He is a Fellow of the Engineering Institute of Canada, the American Society of Mechanical Engineers, and the Canadian Society of Mechanical Engineering.

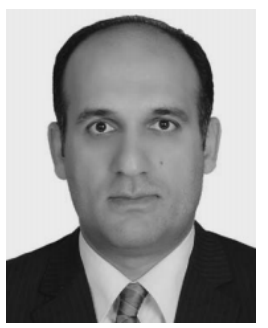

Alireza Kasaiezadeh received the B.Sc. and M.Sc. degrees from Sharif University of Technology, Tehran, Iran, and the Ph.D. degree in mechanical and mechatronic engineering from the University of Waterloo, Waterloo, ON, Canada. He did a two-year postdoctoral fellowship and was a Research Assistant Professor with the University of Waterloo. He is currently a Senior Researcher with GM Technical Center, General Motors Company, Warren, MI, USA, where he is working in the area of automated driving and vehicle control. He has more than 14 years of industrial experience in the automotive industry and, in particular, in active safety systems, chassis control system design, and field testing. His research interests include automated driving, vehicle dynamics, control and optimization.

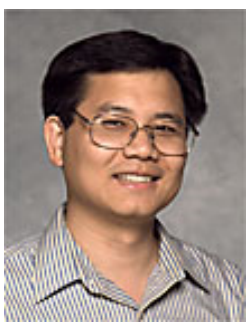

Shih-Ken Chen received the B.S. degree from National Taiwan University, Taipei, Taiwan, in 1985; the M.S. degree in mechanical engineering from the University of WisconsinMadison, Madison, WI, USA, in 1990; and the Ph.D. degree in mechanical engineering from Massachusetts Institute of Technology, Cambridge, MA, USA, in 1996. He then joined the Research and Development Center, General Motors Corporation. He is currently a Staff Researcher with the GM Technical Center, General Motors Company, Warren, MI, USA. His previous work includes collision avoidance systems, electronic stability control, active all-wheel-steer control, and rollover avoidance. His current research interests include holistic chassis and vehicle control for both conventional driveline and electric driveline, driver-in-the-loop vehicle control, and vehicle active safety system and automated driving.

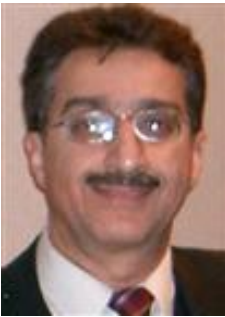

Bakhtiar Litkouhi received the B.Sc. degree in mechanical engineering from Arya-Mehr (Sharif) University of Technology, Iran and the M.Sc. degree in applied mathematics and the Ph.D. degree in systems science, specializing in control, both from Michigan State University, East Lansing, MI, USA. He was an Assistant Professor with Oakland University, Rochester, MI, USA. He has served as the Program Manager for several large-scale projects in automated and intelligent vehicle systems, human machine interface, and integrated vehicle control, where he has made many contributions through numerous patents, publications, and presentations. He is currently the Manager of Automated Driving and Vehicle Control Systems, General Motors Global Research and Development, Warren, MI, USA. In addition to his current responsibilities, he is a board member of the Intelligent Transportation Society of Michigan, a member of the Board of Directors of the Waterloo Center for Automotive Research (WatCAR), and the Program Manager of GMCarnegie Mellon University Autonomous Driving Collaborative Research Lab. 